\title{
ATN-161 Ameliorates Ischemia/Reperfusion-induced Oxidative Stress, Fibro-inflammation, Mitochondrial damage, and Apoptosis-mediated Tight Junction Disruption in bEnd.3 Cells
}

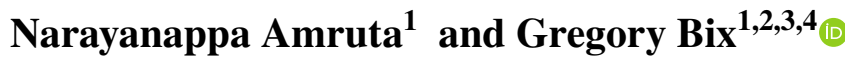

Received 23 March 2021; accepted 27 June 2021

Abstract - We have previously demonstrated the significance of endothelial cell-expressed $\alpha 5 \beta 1$ integrin in ischemic stroke, having shown that $\alpha 5 \beta 1$ integrin endothelial cell-selective knockout mice are significantly resistance to ischemic stroke injury via preservation of the tight junction protein claudin-5 and subsequent stabilization of the blood-brain barrier (BBB). In addition, inhibition of $\alpha 5 \beta 1$ by the small peptide noncompetitive integrin $\alpha 5$ inhibitor, ATN-161, is beneficial in a mouse model of ischemic stroke through reduction of infarct volume, edema, stabilization of the BBB, and reduced inflammation and immune cell infiltration into the brain. In continuation with our previous findings, we have further evaluated the mechanistic role of ATN-161 in vitro and found that oxygen and glucose deprivation and reperfusion (OGD/R)-induced inflammation, oxidative stress, apoptosis, mitochondrial depolarization, and fibrosis attenuate tight junction integrity via induction of $\alpha 5$, NLRP3, p-FAK, and p-AKT signaling in mouse brain endothelial cells. ATN-161 treatment $(10 \mu \mathrm{M})$ effectively inhibited OGD/R-induced extracellular matrix (ECM) deposition by reducing integrin $\alpha 5$, MMP-9, and fibronectin expression, as well as reducing oxidative stress by reducing mitochondrial superoxide radicals, intracellular ROS, inflammation by reducing NLRP3 inflammasome, tight junction loss by reducing claudin-5 and ZO-1 expression levels, mitochondrial damage by inhibiting mitochondrial depolarization, and apoptosis via regulation of p-FAK and p-AKT levels. Taken together, our results further support therapeutically targeting $\alpha 5$ integrin with ATN-161, a safe, well-tolerated, and clinically validated peptide, in ischemic stroke.

KEY WORDS: ATN-161; bEND.3 cells; Fibrosis; Mitochondrial integrity; NLRP3; OGD/R

\footnotetext{
${ }^{1}$ Department of Neurosurgery, Clinical Neuroscience Research Center, Tulane University School of Medicine, Room 1349, 131 S. Robertson, Ste 1300, New Orleans, LA 70112, USA

${ }^{2}$ Department of Neurology, Tulane University School of Medicine, New Orleans, LA 70112, USA

${ }^{3}$ Tulane Brain Institute, Tulane University, New Orleans, LA 70112, USA

${ }^{4}$ To whom correspondence should be addressed at Department of Neurosurgery, Clinical Neuroscience Research Center, Tulane University School of Medicine, Room 1349, 131 S. Robertson, Ste 1300, New Orleans, LA 70112, USA. Email: gbix@tulane.edu
}

\section{INTRODUCTION}

Ischemic stroke, a disruption of blood flow to a part of the brain most commonly due to a blood clot (thrombus) accounts for $\sim 80 \%$ of all strokes and is a significant cause of morbidity and mortality. Intravenous recombinant tissue plasminogen activator (tPA) and/or endovascular thrombectomy are currently used to treat acute 
ischemic stroke $[1,2]$. Both treatments result in recanalization/reperfusion, which, although beneficial, can also result in so-called brain ischemia/reperfusion (I/R) injury. A common cause of $I / R$ injury is the rapid inflow of fluids following an acute/chronic occlusion of blood, nutrients, and oxygen to the tissue triggering the formation of free radicals. Ischemic stroke is followed by blood-brain barrier (BBB) dysfunction and vasogenic brain edema [3]. Structurally, tight junctions (TJs) between brain endothelial cells play an important role in maintaining the integrity of the BBB. I/R injury leads to an inflammatory response that triggers oxidative stress, cellular apoptosis, mitochondrial damage, extracellular matrix (ECM) degradation, with disruption of TJ integrity, and causes BBB breakdown and brain damage. However, the mechanism of $\mathrm{I} / \mathrm{R}$ injury needs extensive investigation to identify effective therapies [4].

The integrin receptor family is a group of cell surface transmembrane glycoprotein receptors for the ECM composed of noncovalently bound $\alpha$ and $\beta$ subunits and expressed throughout the body, including in the vasculature $[5,6]$. Integrins play important roles in cell migration, cellular adhesion, and cellular survivability. One integrin family member, integrin $\alpha 5 \beta 1$, is of vital interest due to its increased expression in hypoxia and stroke models. We and other groups have previously shown that integrin $\alpha 5 \beta 1$ induction and its mediated downstream signaling play a critical role in the pathology of ischemic stroke and brain hypoxia [7-10].

Our laboratory has shown that endothelial cellspecific integrin $\alpha 5 \beta 1$ gene deficiency results in significant resistance to ischemic stroke injury in mice [11]. Furthermore, treatment with a novel $\alpha 5 \beta 1$ integrin inhibitor, the pentapeptide ATN-161, reduces neuroinflammation, stabilizes ECM remodeling and is neuroprotective in murine ischemic stroke and protection from SARS-CoV-2 infection in k18-hACE2 transgenic mice [12,8]. To further elucidate ATN-161's precise therapeutic mechanisms of action, we studied ATN-161 in an in vitro model of stroke, oxygen-glucose deprivation/reoxygenation (OGD/R) in brain endothelial cells. We found that ATN-161 effectively protected endothelial cells against OGD/R induced pathology by inhibiting oxidative stress, inflammation, collapse of mitochondrial membrane potential (MMP), fibrosis, and tight junction loss via its mechanistic inhibition of $\alpha 5 \beta 1$ induced NLRP3 mediated FAK and AKT signaling. Taken together, our results suggest that inhibition of $\alpha 5 \beta 1$ integrin by ATN-161 is a promising novel target for the clinical treatment of ischemic stroke.

\section{MATERIALS AND METHODS}

\section{Cell Culture and Treatments}

Mouse brain microvascular endothelial cells (bEnd.3) were obtained from American Type Culture Collection (ATCC; Manassas, VA, USA). Cells were grown in high-glucose DMEM medium supplemented with $10 \%$ FBS and $1 \%$ penicillin/streptomycin (Thermo Scientific, Rockford, IL, USA) in a $37{ }^{\circ} \mathrm{C}$ humidified incubator with 95\% air and $5 \% \mathrm{CO}_{2}$ (Thermo Fisher Scientific, Waltham, MA, USA). Cells were cultured and multiplied in $75-\mathrm{cm}^{2}$ culture flasks. Cells cultured in 6-well plates were used for western blotting and other cell assays. The cells were cultured in 8 well chamber slides for immunofluorescence staining. Cells were pretreated with freshly prepared ATN-161 (5, 10, 25, and 50 $\mu \mathrm{M})$ (Medkoo Biosciences, Morrisville, $\mathrm{NC}$, following the instructions of the manufacturer) $1 \mathrm{~h}$ before oxygen-glucose deprivation (OGD) and during OGD/reoxygenation (OGD/R).

\section{OGD/R Treatment}

OGD/R was induced in $70 \%$ confluent bEnd. 3 cells by washing cells with fresh medium, which was then replaced with glucose-free DMEM and then incubation with $95 \% \mathrm{~N} 2 / 5 \% \mathrm{CO}_{2}$ for $6 \mathrm{~h}$. Cells were then regluconated and reoxygenated by placing them in fresh normal DMEM and in an incubator with $95 \%$ air $/ 5 \% \mathrm{CO}_{2}$ (normoxia), respectively. Control cells were incubated with normal medium for $6 \mathrm{~h}$, and replaced with normal medium with normoxic conditions in a manner identical to that for OGD/R $[11,13]$.

\section{Mitochondrial Superoxide Estimation}

MitoSOX was measured using the fluorogenic probe MitoSOX Red (Invitrogen, Paisley, UK). In live cells, MitoSOX Red selectively enters mitochondria and emitted red fluorescence when oxidized by superoxide anions. After $6 \mathrm{~h}$, OGD cells were switched to reoxygenation for $24 \mathrm{~h}$ and incubated for $20 \mathrm{~min}$ at $37^{\circ} \mathrm{C}$ with MitoSOX Red $(2.5 \mathrm{mmol} / \mathrm{L})$. Cells were then washed with HBSS to remove excess dye, and fluorescence images were immediately captured using a ZOE Fluorescent Cell Imager (Bio-Rad, CA, USA) under excitation wavelength $510 \mathrm{~nm}$ and emission wavelength $580 \mathrm{~nm}$. MitoSOX Red fluorescence per cell was quantified by Image $\mathrm{J}$ analysis software (version 1.41 ; NIH) $[14,15]$. 


\section{Estimation of Intracellular ROS}

Intracellular levels of reactive oxygen species (ROS) were detected using DCFDA/H2DCFDA. bEnd. 3 cells were loaded with $20 \mu \mathrm{M}$ DCFDA for $20 \mathrm{~min}$ at $37^{\circ} \mathrm{C}$ and washed twice with HBSS. Fluorescence images were immediately captured using a ZOE fluorescent cell imager (Bio-Rad, CA, USA) under excitation wavelength $495 \mathrm{~nm}$ and emission wavelength $529 \mathrm{~nm}[16,17]$. DCFDA green fluorescence per cell was quantified by ImageJ analysis software (version 1.41 ; NIH).

\section{Flow Cytometric Analysis for Oxidative Stress}

Intracellular levels of ROS were further validated by staining the cells with DCFDA (Abcam, MA, USA) and analyzed with a Beckman Coulter Epics Galios flow cytometer with 2 lasers and 8 detectors running Galios software (v1) (Miami, FL, USA) and Kaluza software (version 1.3, Beckman Coulter, Brea, CA, USA) will be used for post acquisition analysis. After OGD 6 h/reoxygenation $24 \mathrm{~h}$, cells were washed with HBSS and loaded with $20 \mu \mathrm{M}$ DCFDA for $20 \mathrm{~min}$ at $37^{\circ} \mathrm{C}$. Next, cells were trypsinized, resuspended, and placed on ice. DCFDA fluorescence was measured using a Beckman Coulter Epics Galios flow cytometer at an excitation/emission wavelengths of $488 / 540 \mathrm{~nm}$, and data analyzed using the Galios software (v1).

\section{Immunoblotting}

The cells were lysed using RIPA buffer (VWR, Matsonford, PA), and protein concentration was measured using the Pierce ${ }^{\mathrm{TM}}$ BCA Protein Assay Kit (Thermo Fisher Scientific, Waltham, MA). Protein samples $(10 \mu \mathrm{g})$ were loaded onto SDS-PAGE gels (BioRad Mini-PROTEAN TGX), electrophoresed at $90 \mathrm{~V}$ and transferred to PVDF membranes using the iBlot 2 dry blotting system (Invitrogen, Paisley, UK). The membranes were blocked with the EveryBlot Blocking Buffer (Bio-Rad, CA, USA) for 15 min and probed with primary antibodies (listed in Supplementary Table 1) at $4{ }^{\circ} \mathrm{C}$ overnight. The membranes were washed thoroughly with PBS doped with $0.05 \%$ Tween $^{\odot} 20$ and incubated with horseradish peroxidase-conjugated secondary antibodies (listed in Supplementary Table 1) for $1 \mathrm{~h}$ at room temperature. Lastly, the membranes were imaged using the ChemiDoc ${ }^{\mathrm{TM}}$ MP imaging system (Bio-Rad, CA, USA). Densities of each band were normalized to those of $\beta$-actin or GAPDH, whereas phosphorylated proteins were normalized to those of total proteins and analyzed by Image $\mathrm{J}$ analysis software (version $1.41 ; \mathrm{NIH}$ ).

\section{Immunocytochemistry}

Immediately following OGD 6 h/reoxygenation 6 or $24 \mathrm{~h}$ with vehicle or ATN-161 treatment, the cells were fixed in $100 \%$ methanol, washed with PBS, and then incubated with peroxiabolish (Biocare Medical, Concord, CA) for 10 min to block endogenous peroxidase. The cells were washed with PBS, blocked with Rodent M Block (Biocare Medical, Concord, CA) for $15 \mathrm{~min}$ at room temperature, and then incubated with primary antibodies (Supplementary Table 2) prepared in DaVinci diluent (Biocare Medical, Concord, CA) overnight at $4{ }^{\circ} \mathrm{C}$. The cells were then washed with PBS four times and incubated with Alexa Fluor 568 or 488 conjugated secondary antibodies (Supplementary Table 2) (Life Technologies; 1:400), for $45 \mathrm{~min}$ at room temperature. After three washes with PBS, cells were mounted using Vectashield containing DAPI (Vector Labs, Burlingame, CA, USA). The images were obtained using a fluorescence microscope (TS2R, Nikon, Tokyo, Japan). The antibody fluorescence positive pixels per cell was quantified using ImageJ analysis software (version 1.41; NIH).

\section{Apoptosis Analysis by Annexin V/PI Staining by Flow Cytometry}

OGD/R-induced apoptosis and the effect of ATN161 in bEnd.3 cells was determined using Annexin V assay. Cells were cultured in 6-well plates at a seeding density of $3.5 \times 10^{5}$ cells per well. After approximately $24 \mathrm{~h}$, cells were subjected to OGD $6 \mathrm{~h} /$ reoxygenation $24 \mathrm{~h}$ with vehicle or ATN-161. After OGD $6 \mathrm{~h} /$ reoxygenation $24 \mathrm{~h}$, cells were stained with Annexin V-FITC and propidium iodide (PI) for $20 \mathrm{~min}$ at $37^{\circ} \mathrm{C}$. The cell population was sorted into live, early apoptotic, late apoptotic, and necrotic cells using Beckman Coulter Epics Galios flow cytometer with 2 lasers and 8 detectors running Galios software (v1) (Miami, FL, USA). The results were calculated as a percent of cells in late apoptosis. 


\section{Measurement of Mitochondrial Depolarization}

bEnd. 3 cells were subjected to OGD 6/reoxygenation $24 \mathrm{~h}$ and ATN-161 treatments as described above. Mitochondrial depolarization was assessed using a JC-1 assay kit (Invitrogen, Paisley, UK). The cells were incubated in the dark upon $\mathrm{JC}-1(10 \mu \mathrm{g} / \mathrm{mL})$ treatment at $37{ }^{\circ} \mathrm{C}$ for $20 \mathrm{~min}$, then washed thrice with HBSS and visualized using a ZOE Fluorescent Cell Imager (BioRad, California, USA). The JC-1 monomer was photographed under excitation wavelength $460 \mathrm{~nm}$ and emission wavelength $530 \mathrm{~nm}$, whereas the JC-1 polymer was photographed under excitation wavelength $520 \mathrm{~nm}$ and emission wavelength $590 \mathrm{~nm}$. Cells exhibiting red (polymeric) fluorescence were considered to have normal mitochondria, while the cells were considered to have depolarized mitochondria if they exhibited green (monomeric) fluorescence.

\section{TEM Assay}

The bEnd. 3 cells were fixed in $2 \%$ glutaraldehyde in $0.2 \mathrm{M}$ phosphate buffer $\mathrm{pH}$ 7.4. The cells were scraped off and centrifuged to a pellet. The cells were embedded in $3 \%$ agarose (Sigma-Aldrich, St. Louis, MO) and put in the same fixative overnight at $4{ }^{\circ} \mathrm{C}$. The cells were then rinsed four times in $0.1 \mathrm{M}$ phosphate buffer $\mathrm{pH} 7.2$ containing $0.02 \mathrm{M}$ glycine over a $12 \mathrm{~h}$ period. The samples were then post-fixed in $2 \%$ osmium tetroxide for $1 \mathrm{~h}$ in the dark, rinsed in deionized water three times, en bloc stained in $1 \%$ uranyl acetate in the dark for $1 \mathrm{~h}$, rinsed in deionized water three times, dehydrated in gradient ethanol and propylene oxide twice, and then infiltrated in propylene oxide: Epon resin series for a $48 \mathrm{~h}$ period and embedded in Epon at $60{ }^{\circ} \mathrm{C}$ for another $48 \mathrm{~h}$. Ultra-thin sections for transmission electron microscopy (TEM) were cut on a Dupont Sorvall MT-2 microtome. TEM sections were mounted on carbon-coated copper grids (EMS FCF150-CU), stained with $2 \%$ uranyl acetate and Reynolds lead citrate, and the ultra-thin sections were visualized with a JEOL JEM-1400, 120-kV transmission electron microscope, and imaged with a Gatan digital camera [18].

\section{Statistical Analysis}

Data are demonstrated as the means \pm SEM and analyzed by one-way ANOVA followed by a Tukey's post hoc test, using GraphPad Prism 8.0. Data significance was determined as $*$ or $\# p<0.05$, ** or $\# \# p<0.01$, *** or $\# \# \# p<0.001$, and $* * * * p<0.0001$. Each experiment was repeated separately three times.

\section{RESULTS}

\section{ATN-161 Treatment Inhibits OGD/R-induced Integrin $\alpha 5$ Receptor in bEnd.3 Cells}

In the present study, we observed that bEnd 3 cells subjected to $6 \mathrm{~h}$ of OGD followed by different reoxygenation time points showed increased expression of integrin $\alpha 5$ (Fig. 1A), with maximum levels of expression reached with OGD alone and OGD with 2-6 h post-reoxygenation (Fig. 1B (i-ii)). Hence, we further evaluated the effect of ATN-161 in bEnd3 cells subjected to $6 \mathrm{~h}$ of OGD, followed by up to $6 \mathrm{~h}$ of reoxygenation (normal glucose and normoxia; Fig. 1C). The cells were pretreated with vehicle or ATN-161 $(5,10,25$, and $50 \mu \mathrm{M})$ for $1 \mathrm{~h}$ before OGD and during OGD $6 \mathrm{~h} / 6 \mathrm{~h}$ reoxygenation. As shown in Fig. 1D (i-ii), western blot analysis revealed that ATN-161 $(10 \mu \mathrm{M})$ treatment significantly inhibited OGD $6 \mathrm{~h} / 6 \mathrm{~h}$ reoxygenation induced $\alpha 5$ upregulation. Further, we measured the $\alpha 5$ cellular expression in bEND. 3 cells upon OGD/R by immunofluorescence and the representative fluorescence images show highly induced $\alpha 5$ in OGD/R challenged cells compared to cells with vehicle or ATN-161 $(10 \mu \mathrm{M})$ treatment (Fig. 1E (i-iii)). ATN-161 $(10 \mu \mathrm{M})$ treatment significantly inhibits OGD/R induced $\alpha 5$ expression (Fig. 1E (iv-v)).

\section{ATN-161 Protects OGD/R-induced NLRP3-mediated Inflammation in bEnd3 Cells}

Since integrins play critical roles in the induction of inflammation [8, 19, 20], we next analyzed the NOD-, LRR-, and pyrin domain-containing protein (NLRP) 3 inflammasome. Western blot analysis showed that OGD/R induces NLRP3 inflammasome expression levels in bEND.3 cells challenged with $6 \mathrm{~h}$ OGD and $24 \mathrm{~h}$ of reoxygenation compared with control cells (Fig. 2A (i-ii)). ATN-161 treatment significantly inhibits $6 \mathrm{~h}$ OGD/24 h reoxygenation induced NLRP3 upregulation (Fig. 2B (i-ii)). Immunofluorescence of NLRP3 in bEND.3 cells shows highly induced NLRP3 expression in OGD/R challenged cells compared to cells with vehicle or ATN-161 treatment (Fig. 2C (i-iii)). ATN161 significantly reduced OGD/R induced NLPR3 inflammasome (Fig. 2C (iv)). The quantification of NLRP3 fluorescence per cell is presented in Fig. $2 \mathrm{C}(\mathrm{v})$. 
A

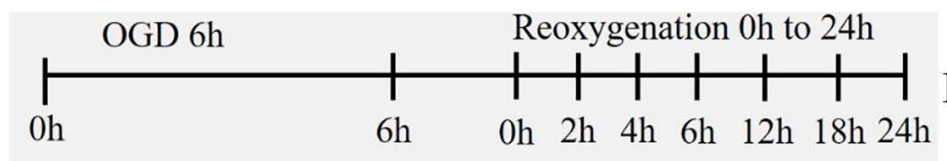

B i

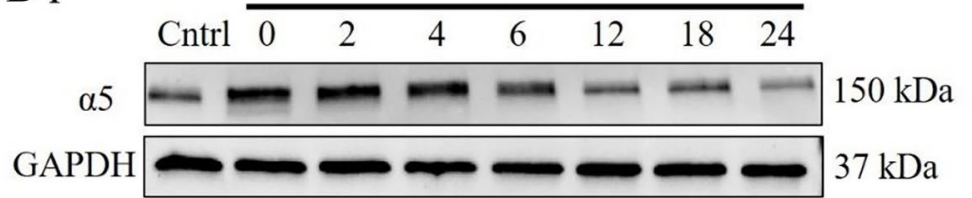

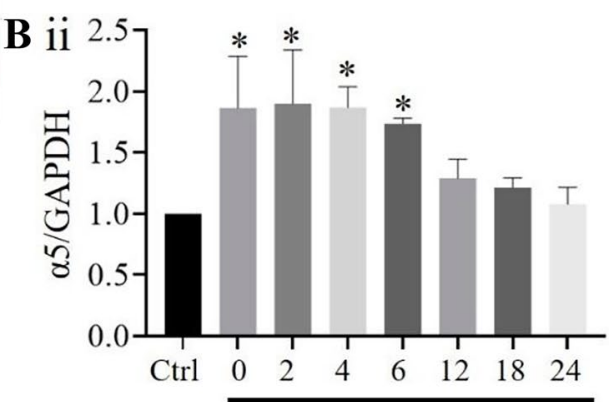

OGD6h/R

C

Vehicle, ATN-161

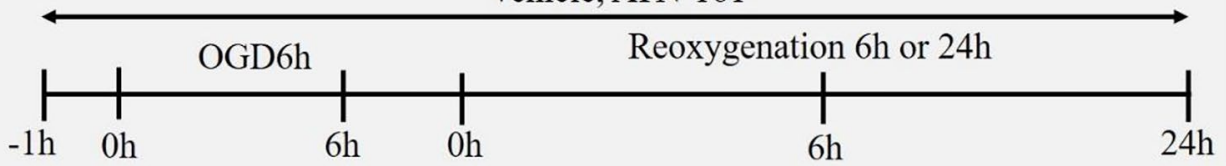

D $\mathrm{i}$

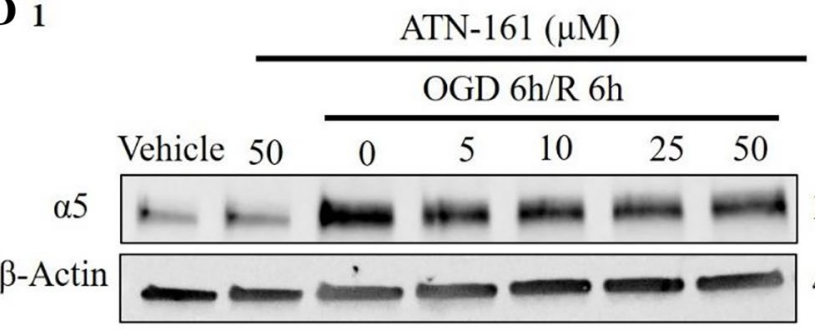

Vehicle

E

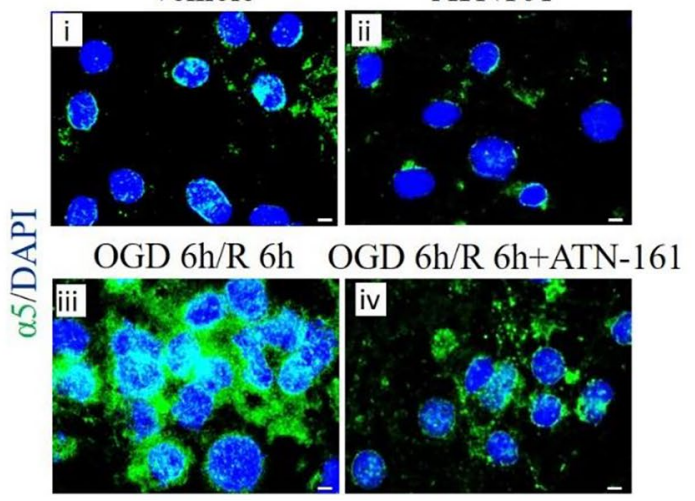

E V
$150 \mathrm{kDa}$

$43 \mathrm{kDa}$
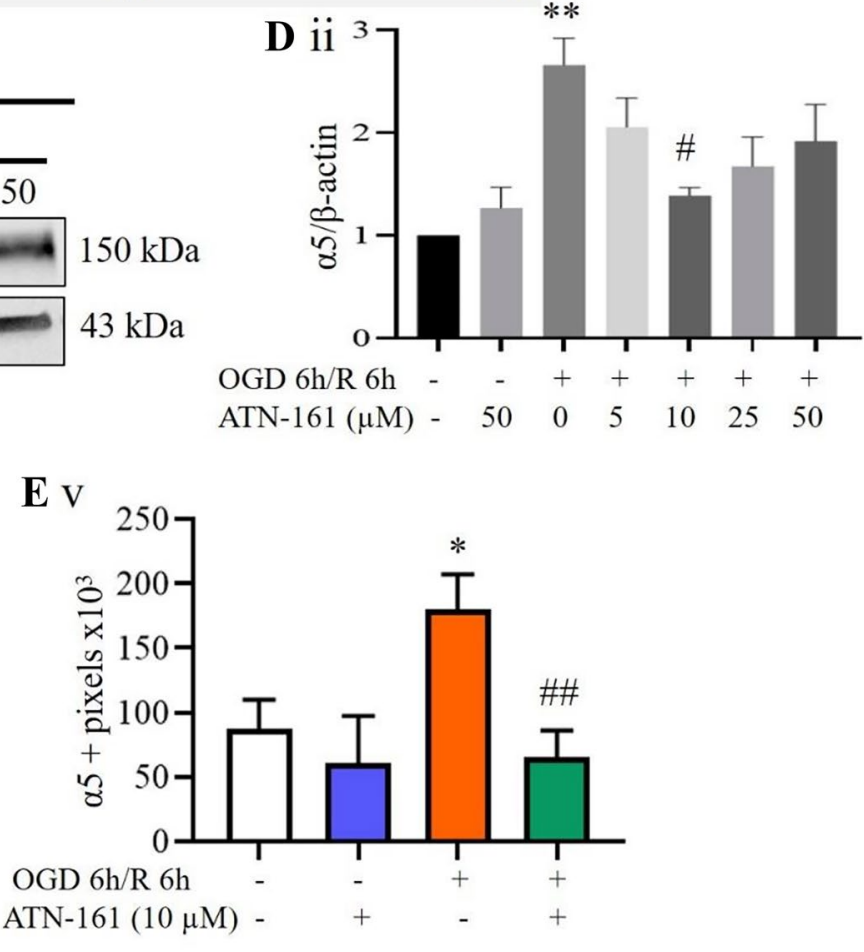

Fig. 1 ATN-161 treatment inhibits OGD/R induced integrin $\alpha 5$ receptor in bEnd. 3 cells. A Experimental protocol of OGD/R in bEnd.3 cells. bEnd.3 cells were subjected to $6 \mathrm{~h}$ of oxygen-glucose deprivation (OGD) followed by different reoxygenation (R) time points as indicated. B (i) Representative western blot images show $\alpha 5$ levels induced during OGD and continued with reoxygenation at 2 , 4 , and $6 \mathrm{~h}$ compared with bEnd.3 cells maintained in normal conditions. B (ii) The immunoblot quantification, as normalized to GAPDH, shows OGD $6 \mathrm{~h}$ and OGD $6 \mathrm{~h}$ with reoxygenation of 2 , 4 , and $6 \mathrm{~h}$ significantly induces $\alpha 5$ upregulation. C Experimental protocol of different doses ATN-161 (5, 10, 25, and $50 \mu \mathrm{M})$ pretreatment in OGD $6 \mathrm{~h} /$ reoxygenation $6 \mathrm{~h}$ or $24 \mathrm{~h}$ challenged bEnd. 3 cells. D (i) The cells were pretreated with vehicle or ATN-161 $(5,10,25$, and $50 \mu \mathrm{M})$ for $1 \mathrm{~h}$ before OGD and during OGD $6 \mathrm{~h} /$ reoxygenation $6 \mathrm{~h}$ and measured for $\alpha 5$ by western blot. D (ii) The immunoblot quantification, as normalized to $\beta$-actin, shows that ATN-161 (10 $\mu$ M) treatment significantly inhibits OGD/R induced $\alpha 5$ upregulation. $\mathbf{E} \alpha 5$ cellular expression in bEnd. 3 cells was measured after OGD 6 h/reoxygenation 6 h by immunofluorescence. $\mathbf{E}$ (i-iii) Representative fluorescence images show highly induced $\alpha 5$ in OGD/R challenged cells compared to cells with vehicle or ATN-161 (10 $\mu \mathrm{M}$ ) treatment. E (iv) ATN-161 treatment significantly inhibits OGD/R induced $\alpha 5$ expression. $\mathbf{E}(\mathrm{v}) \alpha 5$ fluorescence per cell was quantified with ImageJ. Error bars represent the mean \pm SEM from three independent experiments. ${ }^{*} p<0.05, * * p<0.01$ vs control or vehicle; \# $p<0.05$, \#\# $p<0.01$ vs OGD/R; $60 \times$ magnification, scale bar $=10 \mu \mathrm{m}$. 

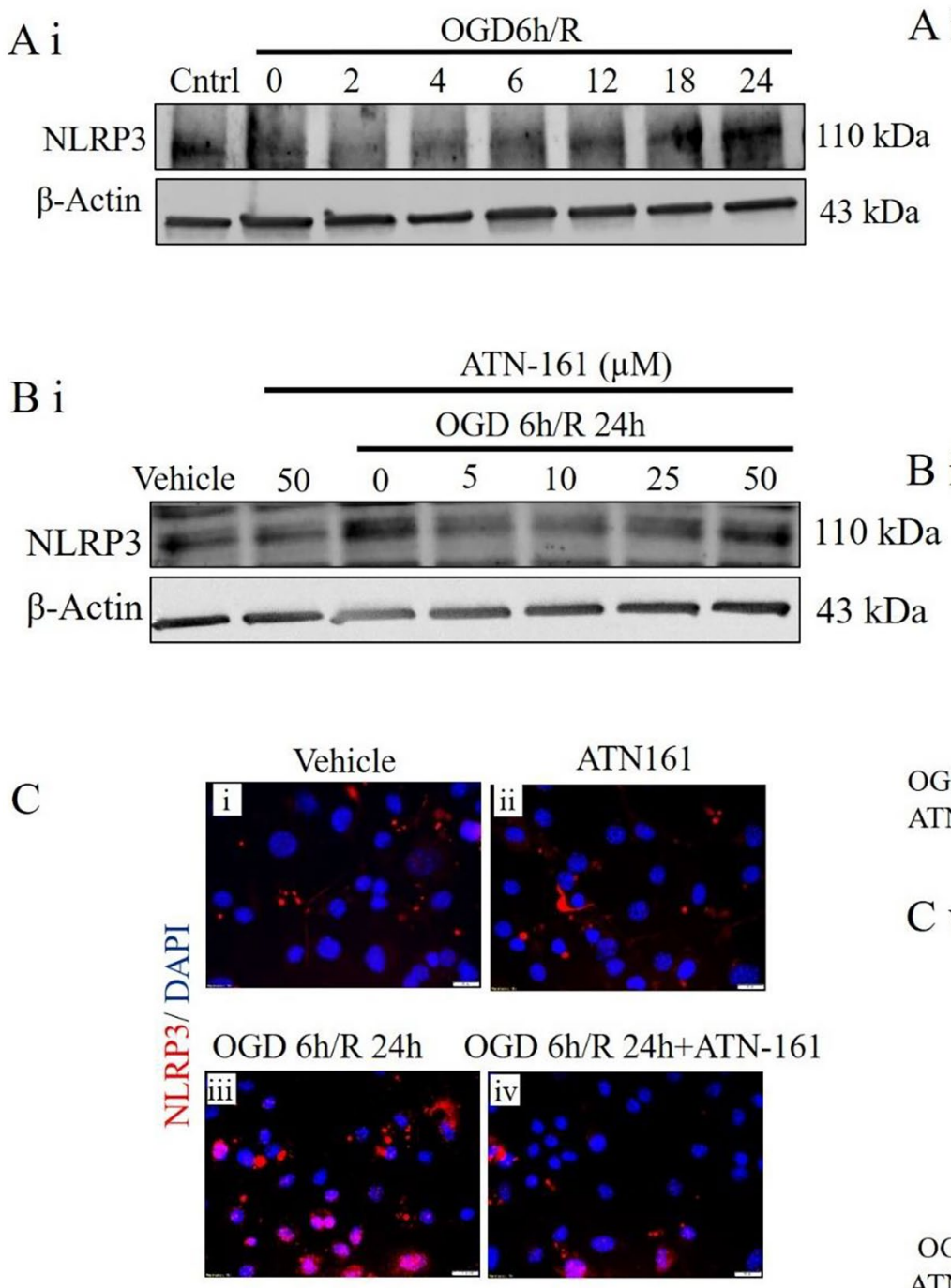
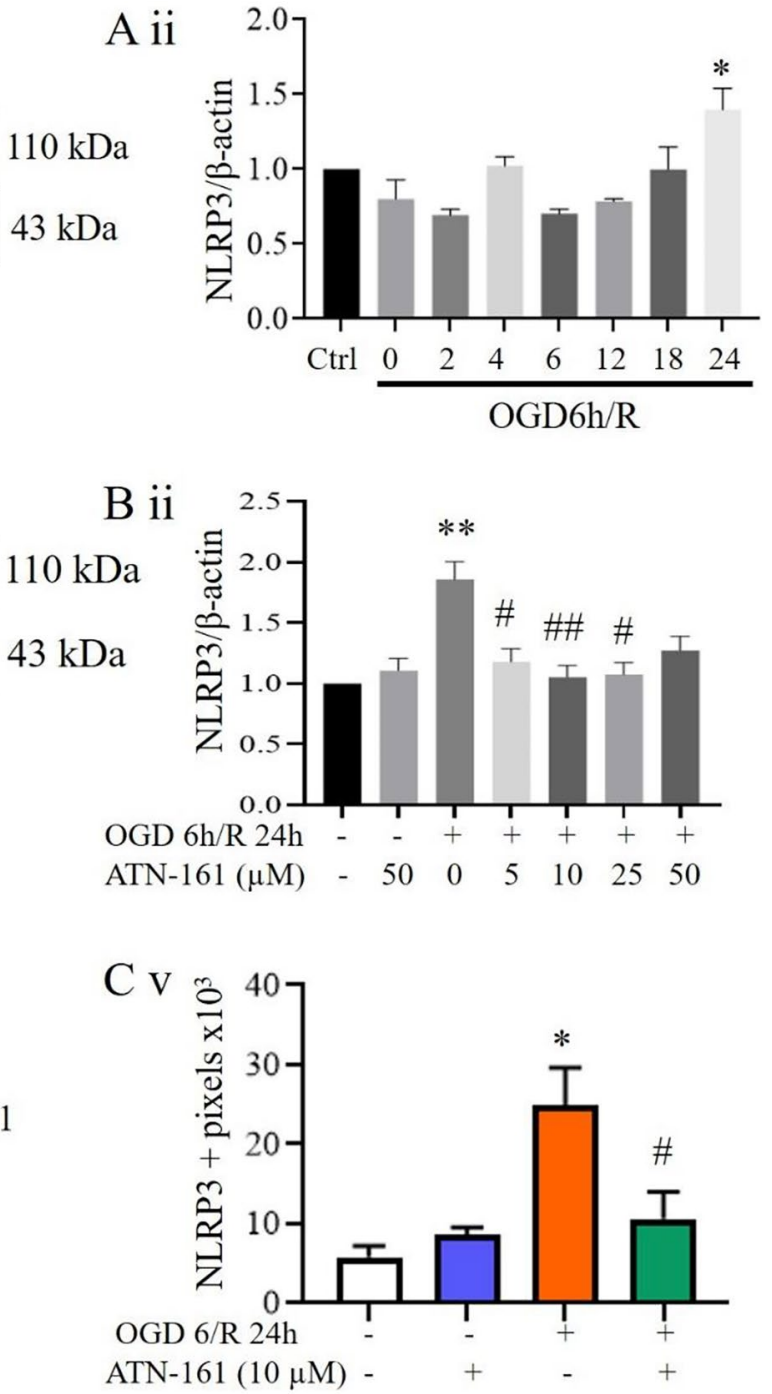

Fig. 2 ATN-161 inhibits OGD/R induced NLRP3 mediated inflammation in bEnd.3 cells. A (i) NLRP3 western blot in 6 h OGD with reoxygenation of $24 \mathrm{~h}$ compared with bEnd.3 cells maintained in normal conditions. A (ii) Quantification of Ai, as normalized to $\beta$-actin, shows OGD $6 \mathrm{~h}$ with $24 \mathrm{~h}$ reoxygenation significantly induces NLRP3 levels. B (i) bEnd.3 cells were pretreated with vehicle or ATN-161 $(5,10,25$, and $50 \mu \mathrm{M})$ for $1 \mathrm{~h}$ before OGD and during OGD $6 \mathrm{~h} /$ reoxygenation $24 \mathrm{~h}$ and measured for NLRP3 by western blot. B (ii) Quantification of B (i) shows that ATN161 treatment at 5,10, and $25 \mu \mathrm{M}$ significantly inhibits OGD/R induced NLRP3 upregulation. C NLRP3 cellular expression in bEnd.3 cells was measured after OGD 6 h/reoxygenation 24 h by immunofluorescence. C (i-iii) Representative fluorescence images show highly induced NLRP3 in OGD/R challenged cells compared to cells with vehicle or ATN-161 $(10 \mu \mathrm{M})$ treatment. C (iv) ATN-161 treatment significantly inhibits OGD/R induced NLRP3 expression. C (v) Quantification of NLRP3 fluorescence per cell. Error bars represent the mean \pm SEM from three independent experiments. * $p<0.05, * * p<0.01$ vs control or vehicle; \# $p<0.05$, \#\# $p<0.01$ vs OGD/R; $40 \times$ magnification, scale bar $=25 \mu \mathrm{m}$.

\section{OGD/R-induced Oxidative Stress Is Inhibited by ATN-161 Treatment in bEnd3 Cells}

$\alpha 5$-induced NLRP3 inflammation generates oxidative stress [21]. Therefore, we further measured oxidative stress by estimation of mitochondrial superoxide radical and intracellular ROS in bEnd. 3 cells. The cells were pretreated with vehicle or ATN-161 $(10 \mu \mathrm{M})$ for $1 \mathrm{~h}$ before OGD and during OGD $6 \mathrm{~h} / 24 \mathrm{~h}$ reoxygenation. Fluorescence microscopy of MitoSOX Red dye (which measures superoxide radicals) showed highly induced superoxide radicals production in OGD/R 

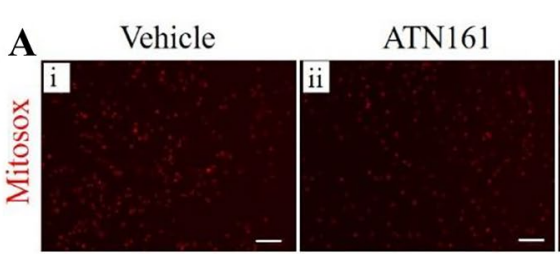

B

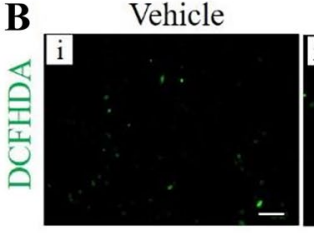

ATN161
OGD6h/R 24h OGD6h/R 24h+ATN-161 A v
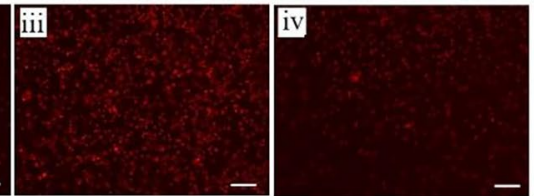

OGD6h/R 24h OGD6h/R 24h+ATN-161 iii

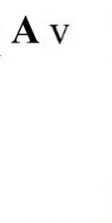

OGD $6 \mathrm{~h} / \mathrm{R} 24 \mathrm{~h}$

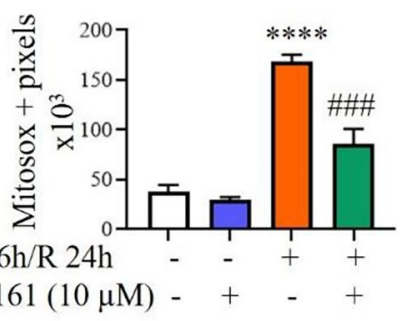

B V
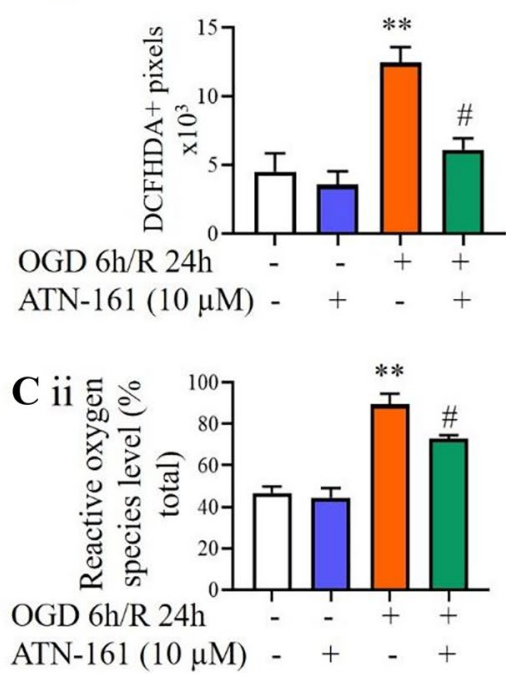

Fig. 3 ATN-161 inhibits OGD/R induced oxidative stress in bEnd.3 cells. bEnd.3 cells were pretreated with vehicle or ATN-161 (10 $\mu$ M) for $1 \mathrm{~h}$ before OGD and during OGD $6 \mathrm{~h} /$ reoxygenation $24 \mathrm{~h}$ and measured for superoxide production and oxidative stress. A (i-iii) Mitochondrial superoxide production in live cells was measured using MitoSOX red dye by fluorescence microscopy, representative fluorescence images show induced superoxide production in OGD/R challenged cells compared to cells with vehicle or ATN-161 treatment. A (iv) ATN-161 treatment inhibits OGD/R induced superoxide production. A (v) MitoSOX Red fluorescence per cell was quantified using the ImageJ software. B (i-iii) Intracellular ROS was measured after OGD/R using DCFH2DA dye by fluorescence microscopy, representative fluorescence images show induced intracellular ROS production in OGD/R challenged cells compared to cells with vehicle or ATN161 treatment. B (iv) ATN-161 treatment inhibits OGD/R induced intracellular ROS production. B (v) DCFH2DA green fluorescence per cell was quantified using the ImageJ software. C Intracellular ROS was further validated after OGD/R using DCFH2DA dye by flowcytometry. C (i) Induced intracellular ROS production in OGD/R challenged cells was noticed with high DCFH2DA-positive cells compared to vehicle or ATN-161 treatment. ATN-161 treatment inhibited ROS production observed by peak shift compared to OGD/R challenged cells. C (ii) DCFH2DA green fluorescence positive cell population measured and presented. Error bars represent the mean \pm SEM from three independent experiments. **** $p<0.0001$, ** $p<0.01$ vs control or vehicle; \# $p<0.05$, \#\#\# $p<0.001$ vs OGD/R; $10 \times$ magnification, scale bar $=100 \mu \mathrm{m}$.

challenged cells compared to cells with vehicle or ATN-161 treatment (Fig. 3A (i-iii)), indicating that ATN-161 treatment significantly inhibits OGD/R induced superoxide production (Fig. 3A (iv-v)). Fluorescence images of intracellular ROS, as measured by DCFH2DA dye, showed highly induced intracellular ROS production in OGD/R challenged cells compared to cells with vehicle or ATN-161 treatment (Fig. 3B (i-iii)). ATN-161 treatment significantly inhibits
OGD/R induced intracellular ROS production (Fig. 3B (iv-v)). This was further validated after OGD/R using DCFH2DA dye by flowcytometry. Induced intracellular ROS production in OGD/R-challenged cells was observed with high DCFH2DA-positive cells compared to vehicle or ATN-161 treatment. ATN-161 treatment significantly inhibited ROS production observed by peak shift compared to OGD/R challenged cells (Fig. 3C (i-ii)). 


\section{ATN-161 Protects Against OGD/R-induced Mitochondrial Damage and Apoptosis in bEnd3 Cells}

As inflammation-induced oxidative stress affects mitochondrial health [22], we next analyzed mitochondrial morphology by TEM analysis. OGD/R-challenged cells showed an increase in mitochondrial size with vacuolations and disorganized cristae, whereas ATN-161 treated cells showed healthier mitochondria with organized cristae (Fig. 4A (i-iv)).

We further analyzed mitochondrial health by measuring the mitochondrial membrane potential (MMP) in bEnd.3 cells upon OGD/R by fluorescence microscopy using the cationic fluorophore JC-1. We noted that more depolarized mitochondria were identified by green fluorescence in OGD/R-challenged cells compared to cells with vehicle or ATN-161 treatment (Fig. 4B (ix-xi)). ATN-161 treatment with OGD/R resulted in fewer depolarized mitochondria (Fig. 4B (xii-xiii)). Since mitochondrial depolarization induces apoptosis, this prompted us to measure apoptosis in bEnd. 3 cells. Annexin V/PI-labeled bEnd.3 cells analyzed by flow cytometry show four quadrants (Fig. 4C (i-iv)) that represent necrotic cells (top left), cells in late apoptotic phase (top right), live cells (bottom left), and cells in early apoptotic phase (bottom right). OGD 6 h/24 h reoxygenation challenge resulted a significant increase in late apoptotic cells (Fig. 4C (i-iii)), whereas ATN161 treatment significantly inhibited the cells undergoing apoptosis (Fig. 4C (iv, v)). Further, OGD 6 h/24 h reoxygenation challenge resulted in a slight increase of early apoptotic cells $(p=0.82)$ and necrotic cells $(p=0.23)$, whereas with ATN-161 $(10 \mu \mathrm{M})$ treatment a slight decrease in OGD/R-induced early apoptotic $(p=0.38)$ and necrotic population was observed $(p=0.39)$. This effect may be due to inhibition of inflammation and oxidative stress.

\section{ATN-161 Attenuates OGD/R-mediated p-FAK and p-AKT Signaling in bEnd3 Cells}

$\alpha 5 \beta 1$ integrin regulates cell survival and proliferation signaling via phosphorylation of AKT and FAK [23, 24]. To understand the role of FAK and AKT signaling, the OGD/R-exposed bEnd.3 cell lysates at each time point were analyzed by western blotting using antibodies specific to phosphorylated forms of FAK and AKT. We determined that p-FAK was induced during OGD $6 \mathrm{~h}$ with reoxygenation at $24 \mathrm{~h}$, whereas p-AKT levels were induced in OGD $6 \mathrm{~h}$ with reoxygenation at $18 \mathrm{~h}$ and $24 \mathrm{~h}$ compared with bEnd. 3 cells maintained in normal condition (Fig. 5A (i) and C (i)). Protein levels were quantified by densitometry and normalized to total FAK/AKT (Fig. 5A (ii) and C (ii)). Furthermore, pretreatment with ATN-161 $(10 \mu \mathrm{M})$ for $1 \mathrm{~h}$ before OGD and during OGD $6 \mathrm{~h} / 24 \mathrm{~h}$ reoxygenation significantly inhibited p-FAK (Fig. 5B (i-ii)), while pretreatment with 5 and $10 \mu \mathrm{M}$ ATN-161 also significantly inhibited OGD/R-induced p-AKT (Fig. 5D (i-ii)).

\section{ATN-161 Inhibits OGD/R-induced Fibrosis in bEnd.3 Cells}

$\alpha 5$ induces fibrosis and ECM deposition in stroke, hypoxia, and ischemia reoxygenation $[8,10,25]$. Hence, we further analyzed fibrosis in OGD/R-challenged bEnd. 3 cells by estimation of fibronectin and MMP-9 levels. Fibronectin levels were significantly induced in $6 \mathrm{~h}$ OGD with 6 and $24 \mathrm{~h}$ reoxygenation (Fig. 6A (i-ii)). ATN-161 $(10 \mu \mathrm{M})$ treatment significantly inhibits OGD/R-induced fibronectin upregulation (Fig. 6B (i-ii)). Immunofluorescence staining was measured for fibronectin and MMP-9 after OGD/R and representative fluorescence images show highly induced fibronectin and MMP-9 in OGD/R treated cells compared to cells with vehicle or ATN-161 treatment (Fig. 6C (i-iii) and D (i-iii)). ATN-161 treatment significantly inhibits OGD/R-induced fibronectin and MMP-9 cellular expression (Fig. 6C (iv-v) and D (iv-v)).

\section{ATN-161 Inhibits OGD/R-induced Tight Junction Loss in bEnd3 Cells}

$\alpha 5 \beta 1$-induced ECM deposition leads to BBB breakdown by tight junction protein loss in vivo [10, $26,27]$. Hence, we further analyzed the tight junction proteins claudin-5 and ZO-1 in OGD/R-treated bEnd.3 cell lysates collected from $6 \mathrm{~h}$ OGD followed by different reoxygenation time points and noted decreased expression of claudin- 5 at $6 \mathrm{~h}$ OGD/6 h reoxygenation whereas ZO-1 loss was observed at various time points of reoxygenation compared with cells in normal condition (Fig. 7A (i-ii) and D (i-ii)). ATN-161 (10 $\mu \mathrm{M})$ treatment significantly attenuates the OGD/R induced claudin-5 and ZO-1 tight junction proteins disruption (Fig. 7B (i-ii) and E (i-ii)). Immunofluorescence staining also confirmed that $\mathrm{OGD} / \mathrm{R}$ reduces levels of 


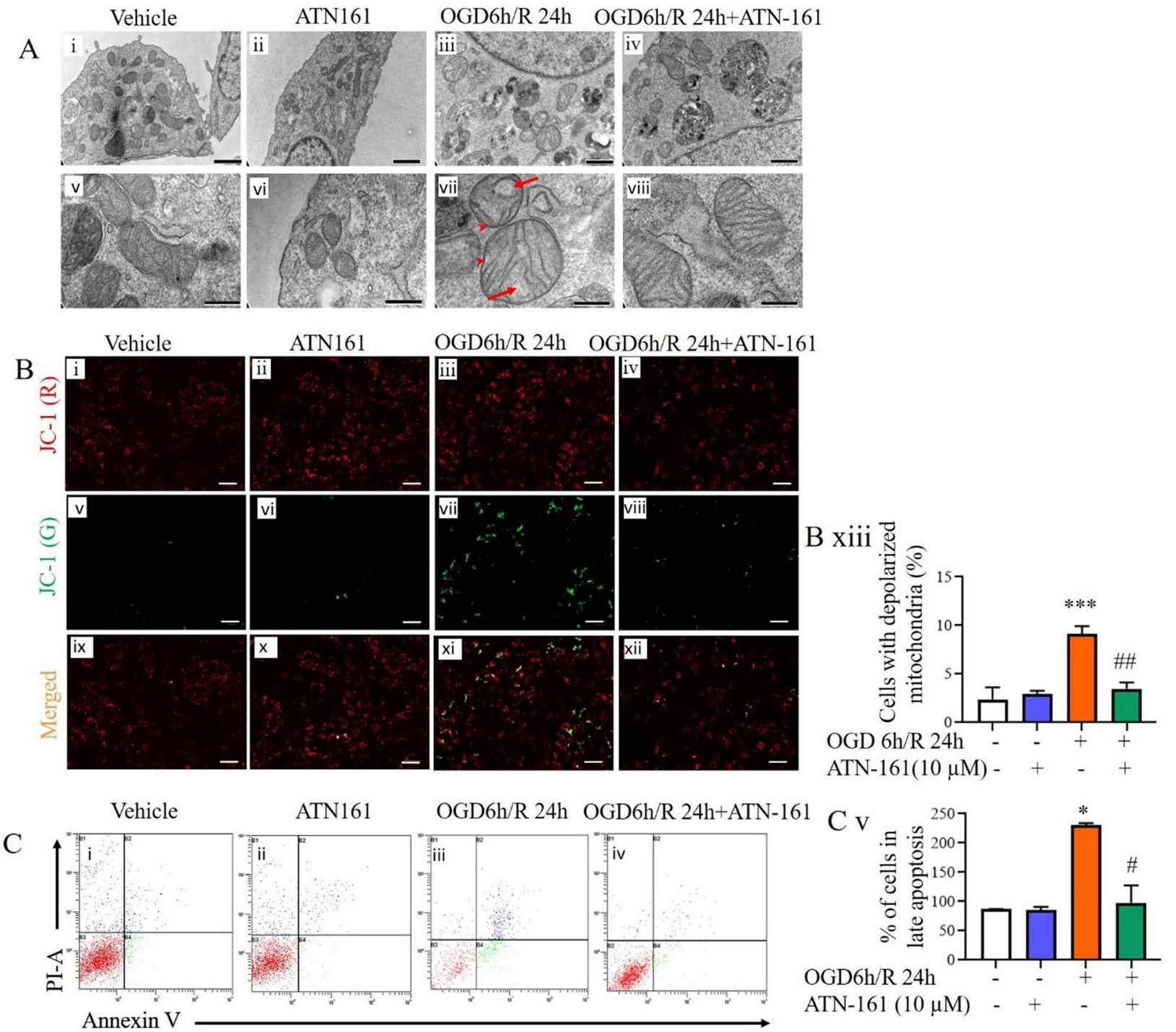

Fig. 4 ATN-161 protects mitochondrial membrane integrity and apoptosis in bEnd.3 cells. bEnd.3 cells were pretreated with vehicle or ATN-161 $(10 \mu \mathrm{M})$ for $1 \mathrm{~h}$ before OGD and during OGD $6 \mathrm{~h} /$ reoxygenation $24 \mathrm{~h}$ and cells harvested and fixed for transmission electron microscopy (TEM), and live cells measured for mitochondrial membrane potential, and apoptosis using annexin V/propidium iodide (PI) labeling by flow cytometric analysis. A Representative TEM images show the presence of abnormal mitochondria following OGD/R insult with increase in mitochondrial size with vacuolations, and disorganized cristae compared with control or ATN-161-treated cells (A, i-iii), whereas ATN-161 treated cells with OGD/R showed healthier mitochondrial with organized cristae (A, iv). Scale bar $=\mathbf{A}$ (i-iv) $0.5 \mu \mathrm{m}$ and A (v-viii) $200 \mathrm{~nm}$. B Mitochondrial depolarization in live cells was measured using JC-1 dye by fluorescence microscopy, representative fluorescence images show cells with more depolarized mitochondria were identified by green fluorescence in OGD/R challenged cells compared to cells with vehicle or ATN-161 treatment (B, ix-xi). B (xii) ATN-161 treatment with OGD/R has less depolarized cells. B (xiii) Cells with depolarized mitochondria with green fluorescence quantified as the percentage of the total cell number using the ImageJ software. C (i-iv) Apoptosis by flow cytometry analysis of cells stained with Annexin V/ PI. Each figure shows four quadrants and quantification data are represented: necrotic (top left), late apoptotic phase (top right), live (bottom left), and early apoptotic phase (bottom right) cell populations. C (v) The quantification of Annexin V/PI staining shows increase in percent of cells in late apoptosis with OGD/R challenged cells compared to control or ATN-161-treated cells, whereas ATN-161 treatment significantly inhibits late apoptosis population with $\mathrm{OGD} / \mathrm{R}$ treatment. Error bars represent the mean \pm SEM from three independent experiments. $* p<0.05$, $* * * p<0.001 \mathrm{vs}$ control or vehicle; $\# p<0.05$, \#\# $p<0.01$ vs OGD/R; $10 \times$ magnification, scale bar $=100 \mu \mathrm{m}$. 

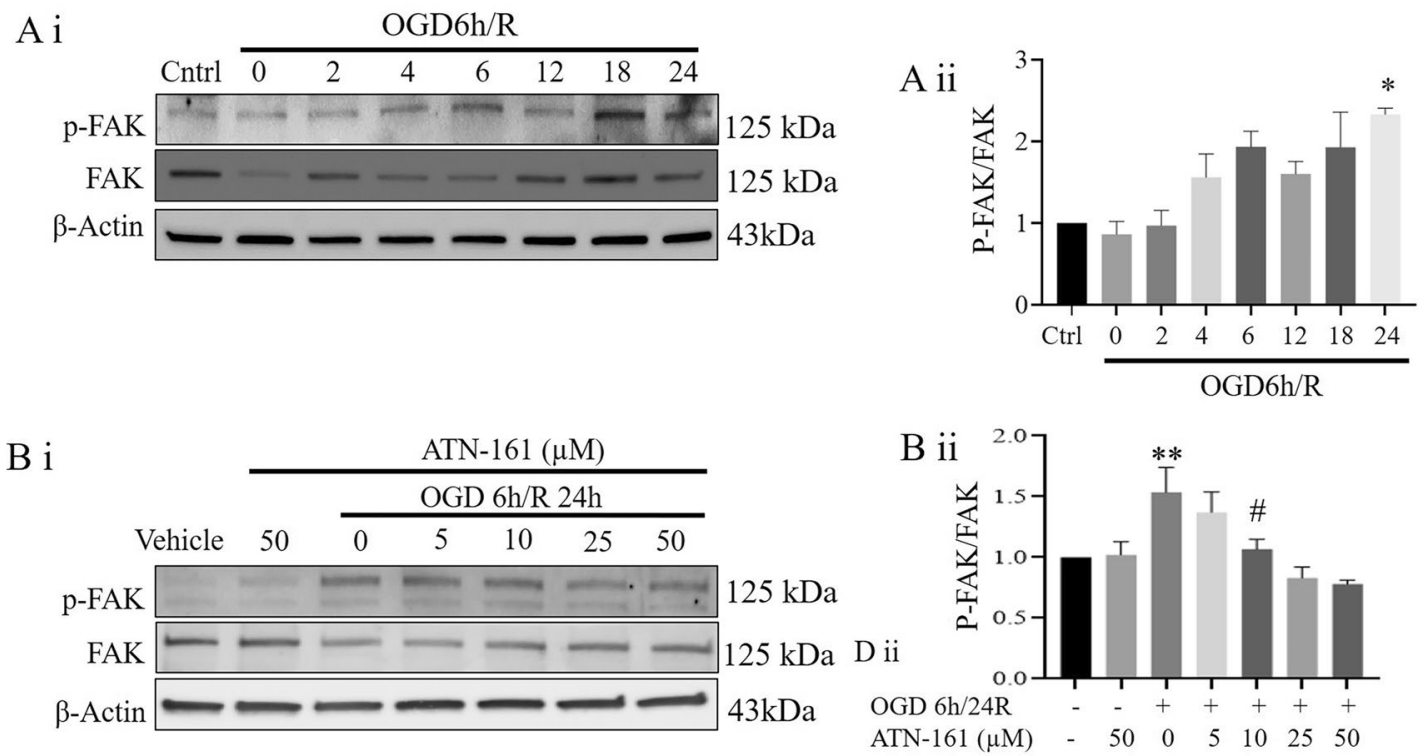

$\mathrm{C}$ i

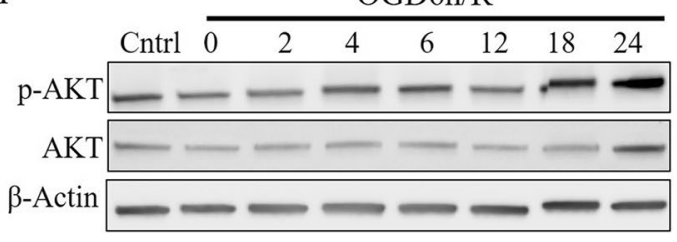

$60 \mathrm{kDa}$

$60 \mathrm{kDa}$ $43 \mathrm{kDa}$

$\mathrm{D}$ i

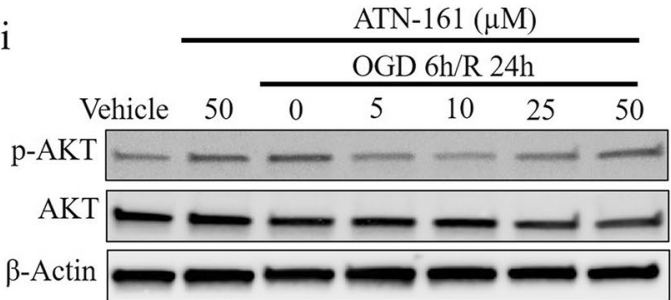

$60 \mathrm{kDa}$

$60 \mathrm{kDa}$

$43 \mathrm{kDa}$
$\mathrm{C}$ ii

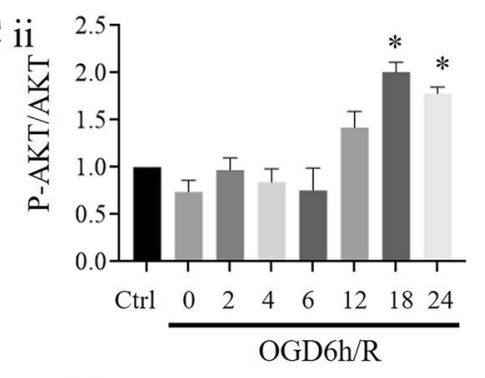

D ii

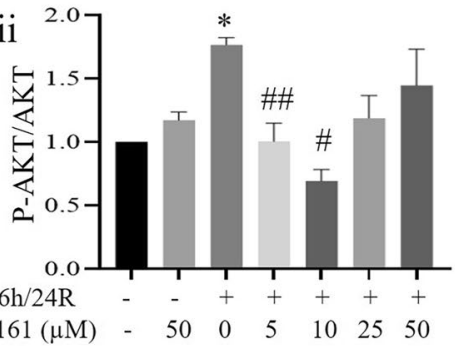

Fig. 5 ATN-161 attenuates OGD/R mediated activation of p-FAK and p-AKT signaling in bEnd.3 cells. bEnd.3 cells were subjected to 6 h of OGD followed by reoxygenation time points. Cell lysates at each time point were analyzed by western blotting using antibodies specific to total protein or phosphorylated form of focal adhesion kinase (FAK) and AKT. A (i) Western blot showing p-FAK levels induced during OGD 6 h with reoxygenation at $24 \mathrm{~h}$ compared with bEnd. 3 cells maintained in normal condition. A (ii) The protein levels were quantified by densitometry and normalized to total FAK that shows significantly induced p-FAK with OGD $6 \mathrm{~h}$ with reoxygenation of $24 \mathrm{~h}$. B (i) The cells were pretreated with vehicle or ATN-161 $(5,10,25$, and $50 \mu \mathrm{M})$ for $1 \mathrm{~h}$ before OGD and during OGD $6 \mathrm{~h} /$ reoxygenation $24 \mathrm{~h}$ and measured for p-FAK by western blot. B (ii) Immunoblot quantification shows that ATN-161 $(10 \mu \mathrm{M})$ treatment significantly inhibits OGD/R induced p-FAK upregulation. C (i) Western blot showing p-AKT levels induced during OGD $6 \mathrm{~h}$ with reoxygenation at $18 \mathrm{~h}$ and $24 \mathrm{~h}$ compared with bEnd. 3 cells maintained in normal condition. C (ii) The protein levels were quantified by densitometry and normalized to total AKT showing that OGD $6 \mathrm{~h}$ with reoxygenation of $18 \mathrm{~h}$ and $24 \mathrm{~h}$ significantly induces p-AKT upregulation. D (i) The cells were pretreated with vehicle or ATN-161 $(5,10,25$, and $50 \mu \mathrm{M})$ for $1 \mathrm{~h}$ before OGD and during OGD $6 \mathrm{~h}$ /reoxygenation $24 \mathrm{~h}$ and measured for p-AKT by western blot. D (ii) Immunoblot quantification shows ATN-161 (5 and $10 \mu \mathrm{M}$ ) treatments significantly inhibit OGD/R induced p-AKT upregulation. Error bars represent the mean \pm SEM from three independent experiments. $* p<0.05, * * p<0.01$ vs control or vehicle; $\# p<0.05$, \#\# $p<0.01$ vs OGD/R. 
$\mathrm{Ai}$

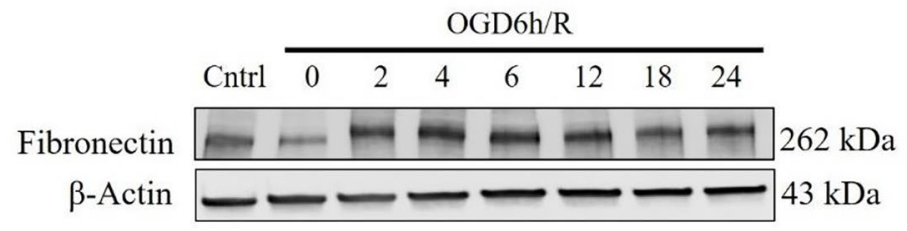

B i

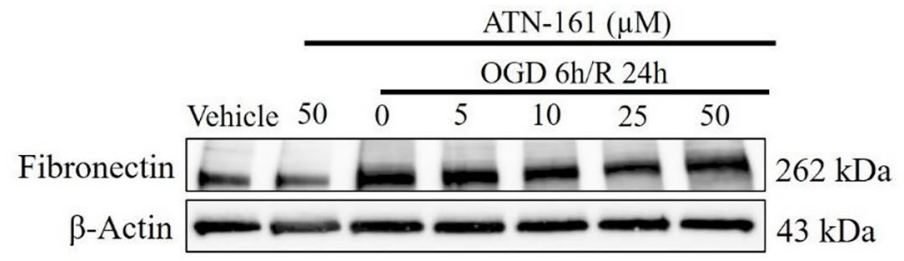

A ii

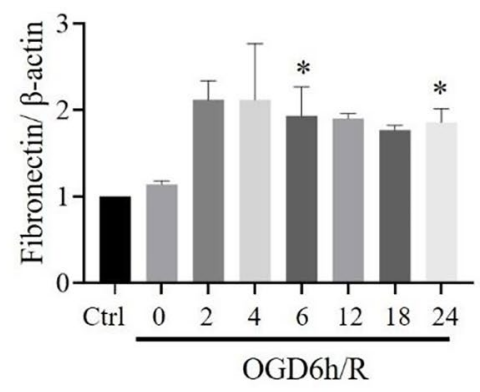

$\mathrm{B}$ ii

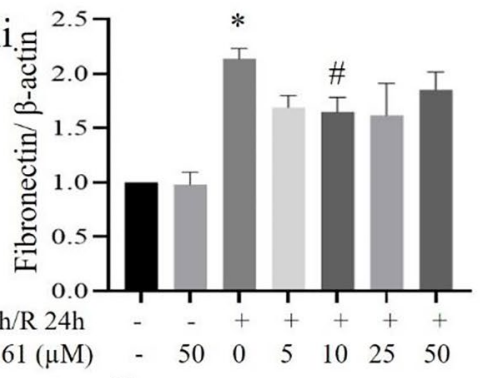

OGD6h/R $24 \mathrm{~h}$

ATN-161 $(\mu \mathrm{M}) \quad-\quad \begin{array}{llllll}50 & 0 & 5 & 10 & 25 & 50\end{array}$

$\mathrm{C}$ V

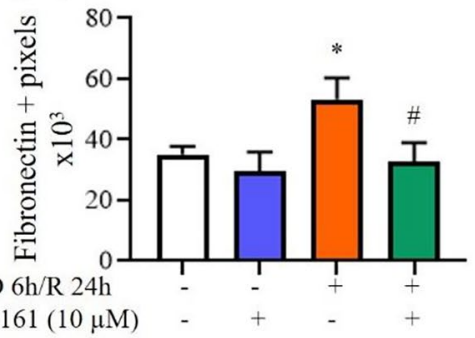

ATN-161 $(10 \mu \mathrm{M})$
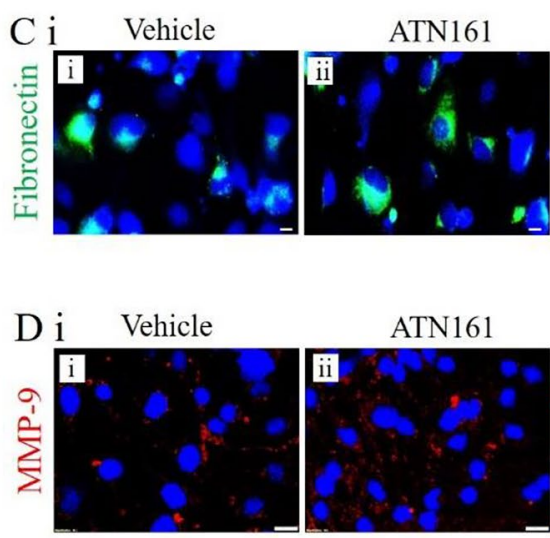

OGD6h/R 24h OGD6h/R 24h+ATN-161

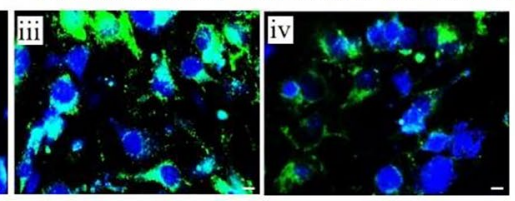

$\mathrm{D} v$

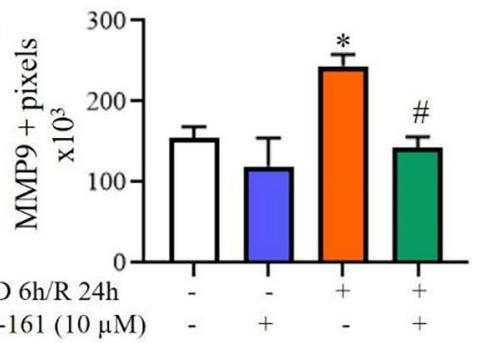

Fig. 6 ATN-161 inhibits OGD/R induced fibrosis in bEnd.3 cells. A (i) Western blot images show fibronectin levels induced in 6 h OGD with reoxygenation of 6 and $24 \mathrm{~h}$ compared with bEnd.3 cells maintained in normal condition. A (ii) Immunoblot quantification shows OGD $6 \mathrm{~h}$ with 6 and $24 \mathrm{~h}$ reoxygenation significantly induces fibronectin levels. B (i) The cells were pretreated with vehicle or ATN-161 $(5,10,25$, and $50 \mu \mathrm{M})$ for $1 \mathrm{~h}$ before OGD and during OGD $6 \mathrm{~h} /$ reoxygenation $24 \mathrm{~h}$ and measured for fibronectin by western blot. B (ii) The immunoblot quantification shows ATN-161 treatment at $10 \mu \mathrm{M}$ significantly inhibits OGD/R induced fibronectin levels. C Fibronectin cellular expression in bEnd.3 cells was measured after OGD 6 h/reoxygenation 24 h by immunofluorescence. C (i-iii) Representative fluorescence images show highly induced fibronectin in OGD/R challenged cells compared to cells with vehicle or ATN-161 $(10 \mu \mathrm{M})$ treatment. C (iv) ATN-161 treatment significantly inhibits $\mathrm{OGD} / \mathrm{R}$ induced fibronectin expression; $60 \times$ magnification, scale bar $=10 \mu \mathrm{m}$. C (v) Quantification of fibronectin fluorescence per cell as in C (iv). D MMP-9 cellular expression in bEnd.3 cells was measured after OGD $6 \mathrm{~h} /$ reoxygenation $24 \mathrm{~h}$ by immunofluorescence. D (i-iii) Representative fluorescence images show highly induced MMP-9 in OGD/R challenged cells compared to cells with vehicle or ATN-161 (10 $\mu$ M) treatment. D (iv) ATN-161 treatment significantly inhibits OGD/R induced MMP-9 expression. D (v) Quantification of MMP-9 fluorescence per cell as in D iv. Error bars represent the mean \pm SEM from three independent experiments. * $p<0.05$ vs control or vehicle; $\# p<0.05$ vs OGD/R; $40 \times$ magnification, scale bar $=25 \mu \mathrm{m}$. 
A $i$

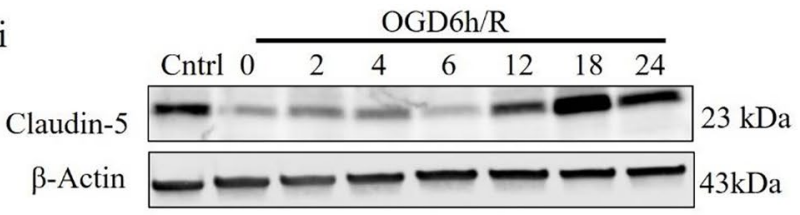

B i

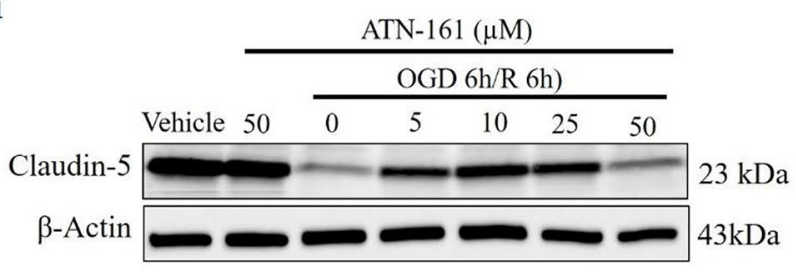

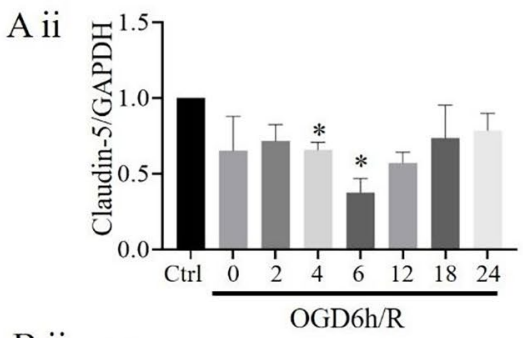

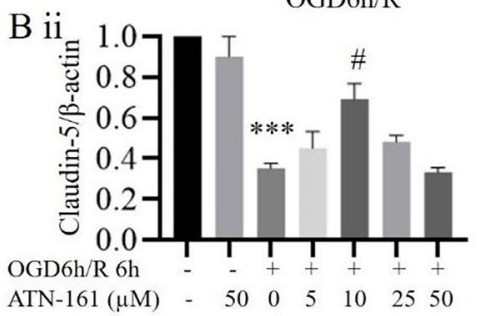

ATN-161 $(\mu \mathrm{M}) \quad-\quad 50 \quad 0 \quad 5 \quad 10 \quad 2550$

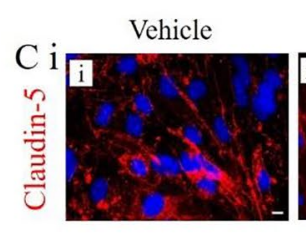

$\mathrm{D}$ i

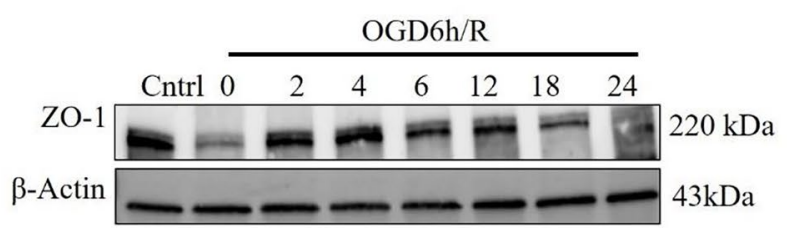

E i

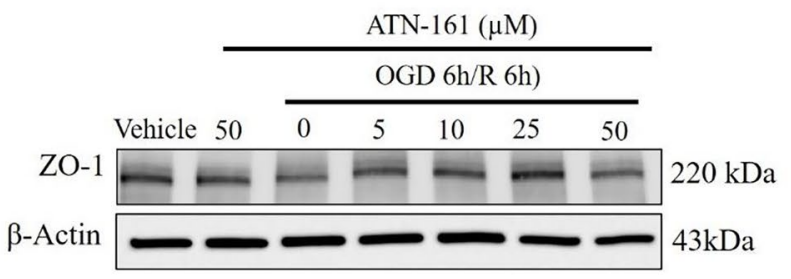

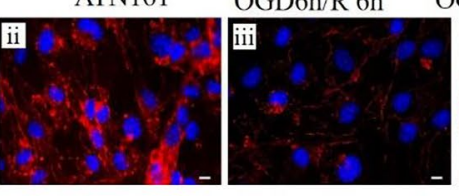

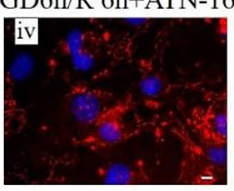

\section{$-$}

C V ATN-161 $(10 \mu \mathrm{M})$ -
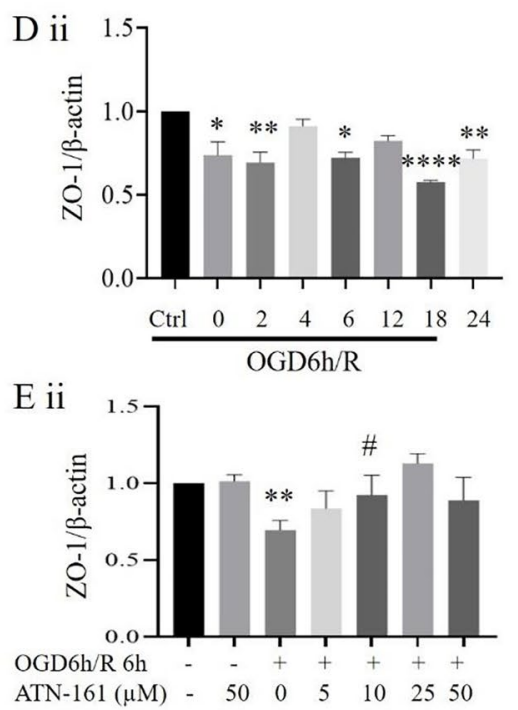

$\operatorname{ATN}-161(\mu \mathrm{M})-\quad \begin{array}{rlllll}50 & 0 & 5 & 10 & 25 & 50\end{array}$

F v
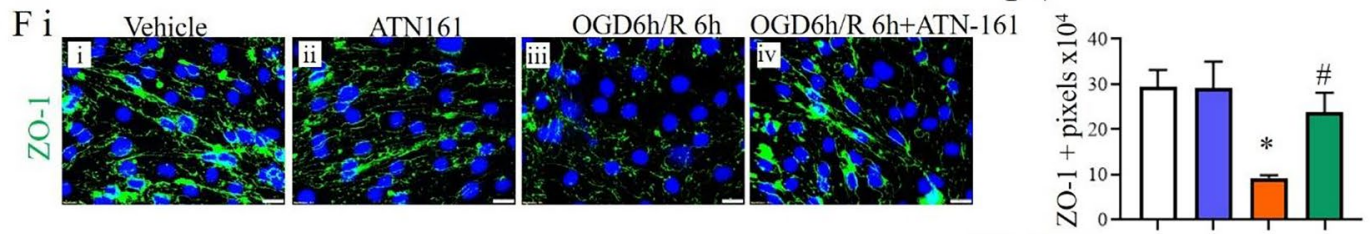

OGD $6 \mathrm{~h} / \mathrm{R} 6 \mathrm{~h}$

ATN-161 $(10 \mu \mathrm{M})$ - 
4 Fig. 7 ATN-161 inhibits OGD/R induced tight junction loss in bEnd.3 cells. Claudin-5 and ZO-1 levels were measured by western blot and fluorescence microscopy in bEnd. 3 cell lysates with OGD $6 \mathrm{~h}$ followed by reoxygenation at different time points and from OGD/R 6 h-ATN161 treatments. A (i) Western blot shows reduced expression of claudin-5 at $6 \mathrm{~h}$ OGD and OGD $6 \mathrm{~h}$ with reoxygenation of 4 and $6 \mathrm{~h}$ compared with bEnd.3 cells maintained in normal condition (fibronectin blot from Fig. 6A was reprobed for claudin-5 in A). A (ii) Immunoblot quantification shows OGD $6 \mathrm{~h}$ with 4 and $6 \mathrm{~h}$ reoxygenation significantly decreased claudin-5 levels. B (i) The cells were pretreated with vehicle or ATN-161 $(5,10,25$, and $50 \mu \mathrm{M})$ for $1 \mathrm{~h}$ before OGD and during OGD $6 \mathrm{~h} /$ reoxygenation $6 \mathrm{~h}$ and measured for claudin-5 by western blot. B (ii) Immunoblot quantification shows ATN-161 treatment at $10 \mu \mathrm{M}$ significantly inhibits OGD/R induced claudin-5 loss. C Claudin-5 cellular expression in bEnd. 3 cells was measured after OGD 6 h/reoxygenation $6 \mathrm{~h}$ by immunofluorescence. C (i-iii) Representative fluorescence images show downregulation of claudin-5 protein levels in OGD/R challenged cells compared to cells with vehicle or ATN-161 $(10 \mu \mathrm{M})$ treatment. C (iv) ATN-161 treatment significantly inhibits OGD/R induced tight junction loss; $60 \times$ magnification, scale bar $=10 \mu \mathrm{m}$. C (v) Claudin-5 fluorescence per cell was quantified using the ImageJ software. D (i) Western blot shows that ZO-1 levels decreased at $6 \mathrm{~h}$ OGD and OGD $6 \mathrm{~h}$ with reoxygenation of 2 , 6,18 , and $24 \mathrm{~h}$ compared with bEnd.3 cells maintained in normal condition. D (ii) Immunoblot quantification shows OGD $6 \mathrm{~h}$ with 2, 6,18 , and 24 h reoxygenation significantly decreased ZO-1 levels. E (i) The cells were pretreated with vehicle or ATN-161 $(5,10,25$, and $50 \mu \mathrm{M}$ ) for $1 \mathrm{~h}$ before OGD and during OGD $6 \mathrm{~h} /$ reoxygenation $6 \mathrm{~h}$ and measured for ZO-1 by western blot. E (ii) Immunoblot quantification shows ATN-161 treatment at $10 \mu \mathrm{M}$ significantly inhibits OGD/R induced ZO-1 loss. F ZO-1 cellular expression in bEnd.3 cells was measured after OGD $6 \mathrm{~h}$ /reoxygenation $6 \mathrm{~h}$ by immunofluorescence. F (i-iii) Representative fluorescence images show downregulation of ZO-1 protein levels in OGD/R challenged cells compared to cells with vehicle or ATN-161 $(10 \mu \mathrm{M})$ treatment. F (iv) ATN-161 treatment significantly inhibits OGD/R induced ZO-1 loss. F (v) ZO-1 fluorescence per cell was quantified using the ImageJ software. Error bars represent the mean \pm SEM from three independent experiments. $* p<0.05$, $* * p<0.01$, *** $p<0.001$, **** $p<0.0001$ vs control or vehicle; \# $p<0.05$, vs OGD/R; $40 \times$ magnification, scale bar $=25 \mu \mathrm{m}$.

tight junction proteins claudin-5 and ZO-1 at OGD/R compared to cells with vehicle or ATN-161 treatment (Fig. 7C (i-iii) and F (i-iii)) whereas ATN-161 treatment significantly inhibited $\mathrm{OGD} / \mathrm{R}$ induced tight junction loss (Fig. 7C (iv-v) and F (iv-v)).

\section{DISCUSSION}

$\mathrm{I} / \mathrm{R}$ injury leads to an inflammatory response that triggers oxidative stress, apoptosis, mitochondrial damage, ECM degradation, and disruption of tight junction integrity, which causes BBB breakdown and brain damage. Structurally, tight junctions between endothelial cells play an important role in maintaining the integrity of the BBB. The BBB comprises brain microvascular endothelial cells that interact with astrocytes, neurons, and pericytes [24, 28-30]. Hence, we used the mouse-derived BMVEC bEnd. 3 cells in this study, a widely used in vitro model for brain endothelial cell signaling in BBB investigations [24, 31].

Recent basic and clinical studies have provided significant progress in our understanding of mechanisms operational in inflammation, oxidative stress, and associated tissue remodeling, including fibrosis, in stroke pathology. However, there remains a great need for a better mechanistic and therapeutic understanding of ECM signaling in I/R injury and stroke pathology. To date, the main therapies for stroke and I/R include tissue plasminogen activator and/or endovascular thrombectomy $[1,2,32,33]$ These treatments remove the blood vesselobstructing thrombus but fail to impact secondary I/R that results from an influx of factors including calcium, cytokines, ROS, growth factors, and matrix metalloproteinases (MMPs) that induce ECM degradation, leukocyte infiltration, and apoptotic cascades. [34, 35]. These processes destabilize the BBB. Although these therapies appear to be effective, not all patients are responsive to treatment. Our preclinical studies show an additional treatment strategy for $\mathrm{I} / \mathrm{R}$ and stroke pathology using the small peptide ATN-161, which has been widely tested in clinical studies in cancer patients [36, 37]. Hence, we further aimed to investigate the mechanistic action of ATN-161 in bEnd.3 cells exposed to OGD/R.

Previous research by Donate et al. showed that ATN161 exhibits a U-shaped dose response curve in vitro and where doses equivalent to $1-10 \mathrm{mg} / \mathrm{kg}$ produced the most significant reduction in activity (angiogenesis and tumor growth) [36]. Another study by Cianfrocca et al. demonstrated in their preclinical efficacy models that ATN-161 exhibited a U-shaped (inverted bell shape) dose-response curve. The study reported the results of a first-in-humans phase 1, dose escalation, pharmacokinetic study of thrice weekly i.v. ATN-161 in patients with advanced cancer. Patients were treated at doses ranging from 0.1 to $16 \mathrm{mg}$ / $\mathrm{kg}$ [37]. Based on these previous researches, in our recent study, we used a concentration of ATN-161 (1 mg/kg) and it showed benefit following experimental ischemic stroke in mice [8]. However, additional stroke dose-response studies should be performed. Likewise, additional studies addressing ATN-161's therapeutic window, and potential long-term effects, need to be conducted.

In the present study, we performed OGD/R in bEnd. 3 cells to mimic I/R injury in vitro. We observed 


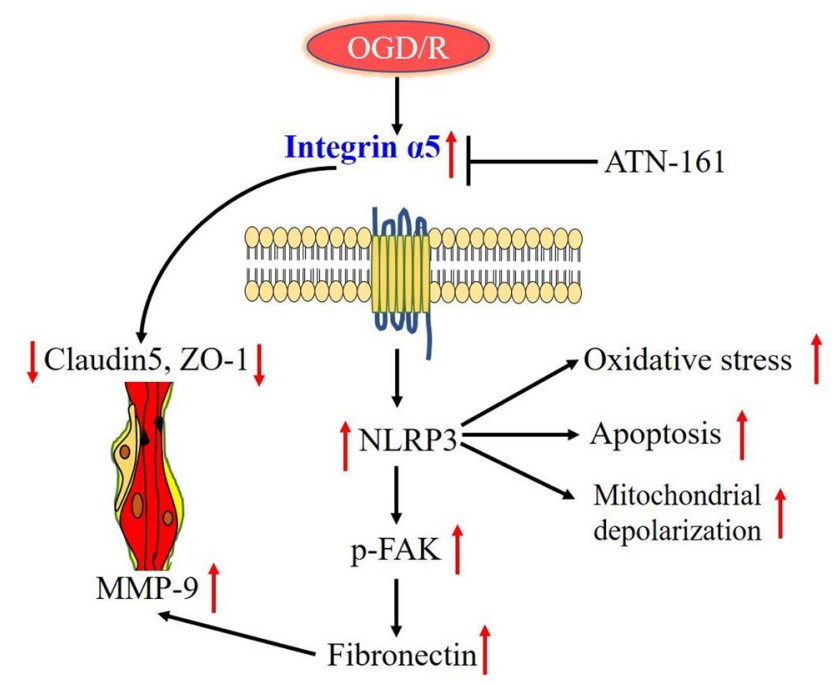

Fig. 8 Proposed mechanism by which ATN-161 ameliorates OGD/R-induced inflammation, oxidative stress, apoptosis, mitochondrial depolarization, and fibrosis mediated tight junction disruption via inhibition of integrin $\alpha 5$, NLRP3, and p-FAK in bEnd. 3 cells.

that (1) OGD/R-induced brain endothelial cell injury via induction of $\alpha 5$, accompanied by the activation of the NLRP3 inflammasome, (2) OGD/R-induced $\alpha 5$ and NLRP3-induced oxidative stress, mitochondrial damage, and apoptosis in bEnd.3 cells. (3) The OGD/Rinduced stress responses further activated p-FAK and p-AKT, induced fibrosis by upregulation of fibronectin and MMP-9, and disruption of tight junctions; and (3) Inhibition of OGD/R induced $\alpha 5$ activation by treatment of bEnd. 3 cells with ATN-161 prevented bEnd. 3 damage by suppressing the up-regulation of $\alpha 5$, NLRP3, and further inhibited oxidative stress, apoptosis, mitochondrial damage, reduced fibrosis by inhibition of fibronectin and MMP-9 induction, and tight junction loss by restoration of claudin-5, and ZO-1, potentially via inhibition of phosphorylation of FAK and AKT proteins. Taken together, our results provide mechanistic evidence that endothelial cell damage is reduced by inhibiting the activation of $\alpha 5$ with ATN-161 during OGD/R.

Previous studies shown that $\alpha 5 \beta 1$ expression was highly upregulated in hypoxia and stroke models $[8,10$, 25]. Our group previously showed that integrin $\alpha 5 \beta 1$ elimination in endothelial-specific knockout ( $\alpha 5 \beta 1$-EC-KO) mice showed resistance to ischemic stroke injury via restoration of tight junction protein claudin- 5 and exhibited minimal to no disruption of the BBB barrier [11]. In another study, we showed that $\alpha 5 \beta 1$ inhibition by ATN-161 in a mouse model of ischemic stroke is beneficial through reduction of brain $\alpha 5 \beta 1$ expression, BBB disruption, $\mathrm{CD} 45^{+}$leukocytes brain extravasation, edema, and fibrosis. In our present study, we similarly observed that OGD/Rinduced $\alpha 5 \beta 1$ protein induction and cellular expression which could also be inhibited by ATN-161 treatment.

Inflammation is a critical mediator in I/R injury, and we previously observed that ATN-161 inhibits I/R-induced IL1 $\beta$, CXCL12, and CD45+ leukocytes [8, 38-40]. In the present study, we observed that OGD/R induced NLRP3 inflammasome induction and cellular expression whereas ATN-161 significantly inhibited OGD/R induced NLRP3 inflammasome levels. Our result is in agreement with a previous study that showed that ATN-161 inhibits VEGFinduced retinal neovascularization in a mouse model by inhibiting integrin $\alpha 5 \beta 1$ and the NLRP3 inflammasome [41]. Another study by Mortimer et al. reported that RGDbinding integrin $\alpha 5 \beta 1$ is required for Entamoeba histolytica (Eh) to activate the NLRP3 inflammasome [42, 43].

Inflammation induces oxidative stress [44], and a previous report shows that $\alpha 5 \beta 1$ lentivirus transduction to rat bone marrow mesenchymal stem cells increase free radical nitric oxide production [45]. Here, we observed that OGD/R induced mitochondrial superoxide generation and intracellular ROS production where as ATN-161 significantly inhibited OGD/R induced superoxide radical and ROS production.

Mitochondria are an important source for ROS, mitochondrial metabolism generated electrons transport diverts to the formation of superoxide [46], and the production of 
ROS accompanied by changes in mitochondrial metabolism effects mitochondrial integrity and, depolarization and induce apoptosis [47, 48]. Therefore, we further analyzed mitochondrial integrity by TEM, which showed that OGD/R challenge resulted in disorganized cristae, with mitochondrial blebbing and vacuolization, whereas ATN161 treatment effectively inhibited OGD/R induced mitochondrial blebbing, vacuolization, and the mitochondria observed with organized cristae. We next analyzed the mitochondrial depolarization by JC-1 immunofluorescence that showed OGD/R induced mitochondrial depolarization and ATN-161 treatment effectively rescued OGD/R induced mitochondrial depolarization. Furthermore, we measured cellular apoptosis by flow cytometry and found that OGD/R increased late apoptotic cell population, which could also be inhibited by ATN-161 treatment. This is in agreement with previous reports that have shown that anti- $\beta 1$ antibodies and arginine-glycine-aspartic acid (RGD) peptide pretreatment decreased apoptosis in isolated pancreatic islets cells [49].

OGD/R-mediated integrin engagement induced inflammation, ROS, and mitochondrial membrane potential and triggered the signal transduction cascade that regulate cell proliferation, differentiation, and death [50]. This was analyzed by measuring the phosphorylation of FAK and AKT proteins that play a role in cell proliferation, differentiation, and death [24, 51, 52]. In the present study, we observed that OGD/R induced FAK, and AKT phosphorylation, which could be inhibited by ATN-161 treatment. In line with our observation, a previous study also demonstrated that OGD/R-induced phosphorylation of both FAK and AKT at a 24-72 h time point could be inhibited by $\alpha 5$ integrin-specific siRNA silencing in bEnd. 3 cells [24].

$\alpha 5 \beta 1$ integrins are adhesion receptors for fibronectin and play a critical role in ECM deposition [10, 53]. In the current study, we measured the fibrosis by immunoblotting of fibronectin, which showed that OGD/R induced fibronectin protein expression, whereas ATN-161 significantly inhibited OGD/R-induced fibronectin expression. Similarly, OGD/R-induced cellular expression of fibronectin is also significantly inhibited by ATN-161 treatment in bEnd. 3 cells. Fibronectin stimulates secretion of MMP-9 by integrin receptor $\alpha 5 \beta 1$, and the blockade of $\alpha 5$ abrogated the fibronectin-mediated stimulation of MMP-9 via inhibition of p-FAK [54]. Hence, we also measured cellular expression of MMP-9 protein by immunofluorescence and found that OGD/R induces MMP-9 levels, whereas ATN-161 treatment effectively inhibited MMP-9 levels in OGD/R induced bEnd.3 cells.
Previous studies showed that ischemic stroke, OGD/R, and hypoxia result in brain endothelial cell tight junction protein loss $[8,10,13]$. Another interesting study also demonstrated that $\alpha 5 \beta 1 / \mathrm{ZO}-1$ complex increases $\alpha 5 \beta 1-$ fibronectin recruitment [55]. This further prompted us to measure the tight junction proteins claudin- 5 and ZO-1 upon OGD/R treatment. Immunoblotting analysis showed downregulation of claudin-5 and ZO-1, whereas ATN-161 restored tight junction loss in bEnd.3 cells. Similarly, immunofluorescence analysis also showed that ATN-161 effectively restored cellular expression of OGD/R-induced claudin-5 and ZO-1 loss in bEnd. 3 cells. The in vitro observation of tight junction proteins restoration upon ATN-161 treatment are in accordance with our previous observation showing that ATN-161 treatment restores ischemic stroke-induced loss of claudin-5 protein levels [8].

\section{CONCLUSION}

Our study identified that OGD/R induced inflammation, oxidative stress, apoptosis, mitochondrial depolarization, and fibrosis mediated tight junction integrity, potentially via induction of integrin $\alpha 5$, NLRP3, p-FAK, and p-AKT signaling in mouse brain endothelial cells. ATN-161 treatment effectively inhibited OGD/R induced ECM deposition by reducing integrin $\alpha 5$, MMP-9 and fibronectin expression, oxidative stress by reducing mitochondrial super oxide radicles, and intracellular ROS, inflammation by reducing NLRP3 inflammasome, tight junction loss by restoring claudin-5 and ZO-1 expression levels, and mitochondrial damage by inhibiting mitochondrial depolarization and apoptosis via regulation of p-FAK and p-AKT levels, as summarized in Fig. 8. Taken together, our results suggest that inhibition of $\alpha 5$ integrin by ATN161 is a promising novel target for the clinical treatment of ischemic stroke. Importantly, ATN-161 was safe and well-tolerated in several phase I and phase II clinical oncology studies, supporting its potential as a "shovel-ready" clinical stroke therapeutic.

\section{SUPPLEMENTARY INFORMATION}

The online version contains supplementary material available at https://doi.org/10.1007/s10753-021-01509-9. 


\section{ACKNOWLEDGEMENTS}

The authors would like to thank Dr. Hemanth Kumar Kandikattu, Pulmonary Medicine, Tulane University, for technical help in experimental design and manuscript editing. Dr. Ying Xiao, Department of Biological Sciences, Louisiana State University, for TEM analysis. Authors are thankful to Alan Tucker, Flow Cytometry and Cell Sorting Core, Tulane University, for help in flow cytometry experiments. The authors are grateful to Dr. Abir Rahman, Dr. Ning Liu, Department of Neurosurgery, Tulane University, for technical help in experiments. The authors appreciate Isabel Murray-Brown, Wesley Chastain, Meshi Paz, Rebecca Solch, Department of Neurosurgery at Tulane University for assistance. The authors appreciate Loula Burton for help in English correction of the manuscript.

\section{AUTHOR CONTRIBUTION}

NA designed and performed experiments, analyzed the data, and wrote the manuscript. G.J.B. revised and finalized the manuscript. All authors read and approved the final manuscript.

\section{FUNDING}

This work was funded by the National Institutes of Health R01 NS065842 (GB). This research was supported in whole or in part by the Louisiana Board of Regents Endowed Chairs for Eminent Scholars program.

\section{AVAILABILITY OF DATA AND MATERIAL}

The data used to support the findings of this study are available from the corresponding author upon request.

\section{Declarations}

Conflict of Interest The authors declare no competing interests.

Consent for Publication Not applicable.

Conflict of Interest The authors declare that they have no conflict of interest.

\section{REFERENCES}

1. Albers, G.W., Goldstein, L.B., Hess, D.C., Wechsler, L.R., Furie, K.L., Gorelick, P.B., Hurn, P., Liebeskind, D.S., Nogueira, R.G., Saver, J.L. and STAIR VII Consortium. 2011. Stroke Treatment Academic Industry Roundtable (STAIR) recommendations for maximizing the use of intravenous thrombolytics and expanding treatment options with intra-arterial and neuroprotective therapies. Stroke 42 (9): 2645-2650.
2. Albers, G.W., M.P. Marks, S. Kemp, S. Christensen, J.P. Tsai, S. Ortega-Gutierrez, R.A. McTaggart, M.T. Torbey, M. Kim-Tenser, T. Leslie-Mazwi, and A. Sarraj. 2018. Thrombectomy for stroke at 6 to 16 hours with selection by perfusion imaging. New England Journal of Medicine 378 (8): 708-718.

3. Alluri, H., C. Anasooya Shaji, M.L. Davis, and B. Tharakan. 2015. Oxygen-glucose deprivation and reoxygenation as an in vitro ischemia-reperfusion injury model for studying blood-brain barrier dysfunction. Journal of Visualized Experiments 99: e52699. https://doi.org/10.3791/52699.

4. Sun, M.S., H. Jin, X. Sun, S. Huang, F.L. Zhang, Z.N. Guo, and Y. Yang. 2018. Free Radical Damage in Ischemia-Reperfusion Injury: An Obstacle in Acute Ischemic Stroke after Revascularization Therapy. Oxidative medicine and cellular longevity. https:// doi.org/10.1155/2018/3804979.

5. Pulous, F.E., and B.G. Petrich. 2019. Integrin-dependent regulation of the endothelial barrier. Tissue Barriers 7 (4): 1685844. https://doi.org/10.1080/21688370.2019.1685844.

6. van der Flier, A., K. Badu-Nkansah, C.A. Whittaker, D. Crowley, R.T. Bronson, A. Lacy-Hulbert, and R.O. Hynes. 2010. Endothelial $\alpha 5$ and $\alpha$ v integrins cooperate in remodeling of the vasculature during development. Development 137 (14): 2439-2449. https:// doi.org/10.1242/dev.049551.

7. Arimoto-Ishida, E., M. Sakata, K. Sawada, M. Nakayama, F. Nishimoto, S. Mabuchi, T. Takeda, T. Yamamoto, A. Isobe, Y. Okamoto, and E. Lengyel. 2009. Up-regulation of $\alpha 5$-integrin by E-cadherin loss in hypoxia and its key role in the migration of extravillous trophoblast cells during early implantation. Endocrinology 150 (9): 4306-4315. https://doi.org/10.1210/en.2008-1662.

8. Edwards, D.N., K. Salmeron, D.E. Lukins, A.L. Trout, J.F. Fraser, and G.J. Bix. 2020. Integrin $\alpha 5 \beta 1$ inhibition by ATN-161 reduces neuroinflammation and is neuroprotective in ischemic stroke. Journal of Cerebral Blood Flow \& Metabolism 40 (8): 1695-1708.

9. Li, L., J. Welser-Alves, A. van der Flier, A. Boroujerdi, R.O. Hynes, and R. Milner. 2012. An angiogenic role for the $\alpha 5 \beta 1$ integrin in promoting endothelial cell proliferation during cerebral hypoxia. Experimental neurology 237 (1): 46-54.

10. Milner, R., S. Hung, B. Erokwu, P. Dore-Duffy, J.C. LaManna, and G.J. del Zoppo. 2008. Increased expression of fibronectin and the $\alpha 5 \beta 1$ integrin in angiogenic cerebral blood vessels of mice subject to hypobaric hypoxia. Molecular and Cellular Neuroscience 38 (1): 43-52. https://doi.org/10.1016/j.mcn.2008.01.013.

11. Roberts, J., L. de Hoog, and G.J. Bix. 2017. Mice deficient in endothelial $\alpha 5$ integrin are profoundly resistant to experimental ischemic stroke. Journal of Cerebral Blood Flow \& Metabolism 37 (1): 85-96. https://doi.org/10.1177/0271678X15616979.

12. Narayanappa, Amruta Elizabeth B., Engler-Chiurazzi Isabel C., Murray-Brown Timothy E., Gressett Ifechukwude J., Biose Wesley H., Chastain Jaime B., Befeler Gregory, Bix (2021) In Vivo protection from SARS-CoV-2 infection by ATN-161 in k18-hACE2 transgenic mice. Life Sciences 284119881-10.1016/j.lfs.2021.119881

13. Nan, D., H. Jin, J. Deng, W. Yu, R. Liu, W. Sun, and Y. Huang. 2019. Cilostazol ameliorates ischemia/reperfusion-induced tight junction disruption in brain endothelial cells by inhibiting endoplasmic reticulum stress. The FASEB Journal 33 (9): 10152-10164.

14. Kumar, K.H., A. Tamatam, A. Pal, and F. Khanum. 2013. Neuroprotective effects of Cyperus rotundus on SIN-1 induced nitric oxide generation and protein nitration: Ameliorative effect against apoptosis mediated neuronal cell damage. Neurotoxicology 34: $150-159$.

15. Liao, L.X., M.B. Zhao, X. Dong, Y. Jiang, K.W. Zeng, and P.F. Tu. 2016. TDB protects vascular endothelial cells against oxygen-glucose 
deprivation/reperfusion-induced injury by targeting miR-34a to increase Bcl-2 expression. Scientific reports 6 (1): 1-13.

16. Venuprasad, M.P., K.H. Kumar, and F. Khanum. 2013. Neuroprotective effects of hydroalcoholic extract of Ocimum sanctum against $\mathrm{H} 2 \mathrm{O} 2$ induced neuronal cell damage in SH-SY5Y cells via its antioxidative defence mechanism. Neurochemical research 38 (10): 2190-2200.

17. Kumar, K.H., Venuprasad, M.P., Jayashree, G.V., Rachitha, P., Krupashree, K., Pal, A. and Khanum, F., 2015. Celastrus paniculatus Willd. mitigates t-BHP induced oxidative and apoptotic damage in C2C12 murine muscle cells. Cytotechnology 67(6):955-67.

18. Kumar, K.H., and F. Khanum. 2013. Hydroalcoholic extract of Cyperus rotundus ameliorates $\mathrm{H} 2 \mathrm{O}$ 2-induced human neuronal cell damage via its anti-oxidative and anti-apoptotic machinery. Cellular and molecular neurobiology 33 (1): 5-17.

19. Mezu-Ndubuisi, O.J. and Maheshwari, A. 2020. The role of integrins in inflammation and angiogenesis. Pediatric research 1-8.

20. Park, E.J., Y. Yuki, H. Kiyono, and M. Shimaoka. 2015. Structural basis of blocking integrin activation and deactivation for anti-inflammation. Journal of biomedical science 22 (1): 1-9.

21. Al-Yafeai, Z., A. Yurdagul Jr., J.M. Peretik, M. Alfaidi, P.A. Murphy, and A.W. Orr. 2018. Endothelial FN (Fibronectin) deposition by $\alpha 5 \beta 1$ integrins drives atherogenic inflammation. Arteriosclerosis, thrombosis, and vascular biology 38 (11): 2601-2614.

22. Sun, S., F. Hu, J. Wu, and S. Zhang. 2017. Cannabidiol attenuates OGD/R-induced damage by enhancing mitochondrial bioenergetics and modulating glucose metabolism via pentose-phosphate pathway in hippocampal neurons. Redox biology 11: 577-585.

23. Morozevich, G.E., N.I. Kozlova, N.A. Ushakova, M.E. Preobrazhenskaya, and A.E. Berman. 2012. Integrin $\alpha 5 \beta 1$ simultaneously controls EGFRdependent proliferation and Akt-dependent pro-survival signaling in epidermoid carcinoma cells. Aging (Albany NY) 4 (5): 368-374. https:// doi.org/10.18632/aging.100457.

24. Pang, D., L. Wang, J. Dong, X. Lai, Q. Huang, R. Milner, and L. Li. 2018. Integrin $\alpha 5 \beta 1$-Ang $1 /$ Tie 2 receptor cross-talk regulates brain endothelial cell responses following cerebral ischemia. Experimental \& molecular medicine 50 (9): 1-12.

25. Li, L., F. Liu, J.V. Welser-Alves, L.D. McCullough, and R. Milner. 2012. Upregulation of fibronectin and the $\alpha 5 \beta 1$ and $\alpha v \beta 3$ integrins on blood vessels within the cerebral ischemic penumbra. Experimental neurology 233 (1): 283-291.

26. Baeten, K.M., and K. Akassoglou. 2011. Extracellular matrix and matrix receptors in blood-brain barrier formation and stroke. Developmental neurobiology 71 (11): 1018-1039. https://doi.org/ 10.1002/dneu.20954.

27. Milner, R., S.J. Crocker, S. Hung, X. Wang, R.F. Frausto, and G.J. del Zoppo. 2007. Fibronectin-and vitronectin-induced microglial activation and matrix metalloproteinase-9 expression is mediated by integrins $\alpha 5 \beta 1$ and $\alpha \mathrm{v} \beta 5$. The Journal of Immunology 178 (12): $8158-8167$.

28. Gastfriend, B.D., S.P. Palecek, and E.V. Shusta. 2018. Modeling the blood-brain barrier: Beyond the endothelial cells. Current opinion in biomedical engineering 5: 6-12. https://doi.org/10. 1016/j.cobme.2017.11.002.

29. Roberts, J., M.P. Kahle, and G.J. Bix. 2012. Perlecan and the bloodbrain barrier: Beneficial proteolysis? Frontiers in pharmacology 3: 155.

30. Tajes, M., E. Ramos-Fernández, X. Weng-Jiang, M. Bosch-Morato, B. Guivernau, A. Eraso-Pichot, B. Salvador, X. Fernandez-Busquets, J. Roquer, and F.J. Munoz. 2014. The blood-brain barrier: Structure, function and therapeutic approaches to cross it. Molecular membrane biology 31 (5): 152-167.
31. Watanabe, T., Dohgu, S., Takata, F., Nishioku, T., Nakashima, A., Futagami, K., Yamauchi, A. and Kataoka, Y. 2013. Paracellular barrier and tight junction protein expression in the immortalized brain endothelial cell lines bEND. 3, bEND. 5 and mouse brain endothelial cell 4. Biological and Pharmaceutical Bulletin 36(3):492-5.

32. Benjamin, E.J., S.S. Virani, C.W. Callaway, A.M. Chamberlain, A.R. Chang, S. Cheng, S.E. Chiuve, M. Cushman, F.N. Delling, R. Deo, and S.D. de Ferranti. 2018. Heart disease and stroke statistics—2018 update: A report from the American Heart Association. Circulation 137 (12): e67-e492.

33. Mendez, A.A., Samaniego, E.A., Sheth, S.A., Dandapat, S., Hasan, D.M., Limaye, K.S., Hindman, B.J., Derdeyn, C.P. and OrtegaGutierrez, S., 2018. Update in the early management and reperfusion strategies of patients with acute ischemic stroke. Critical care research and practice 2018.

34. Sandoval, K.E., and K.A. Witt. 2008. Blood-brain barrier tight junction permeability and ischemic stroke. Neurobiology of disease 32 (2): 200-219.

35. Wang, Q., X.N. Tang, and M.A. Yenari. 2007. The inflammatory response in stroke. Journal of neuroimmunology 184 (1-2): 53-68.

36. Doñate, F., G.C. Parry, Y. Shaked, H. Hensley, X. Guan, I. Beck, Z. Tel-Tsur, M.L. Plunkett, M. Manuia, D.E. Shaw, and R.S. Kerbel. 2008. Pharmacology of the novel antiangiogenic peptide ATN161 (Ac-PHSCN-NH2): Observation of a U-shaped dose-response curve in several preclinical models of angiogenesis and tumor growth. Clinical Cancer Research 14 (7): 2137-2144.

37. Cianfrocca, M.E., K.A. Kimmel, J. Gallo, T. Cardoso, M.M. Brown, G. Hudes, N. Lewis, L. Weiner, G.N. Lam, S.C. Brown, and D.E. Shaw. 2006. Phase 1 trial of the antiangiogenic peptide ATN-161 (Ac-PHSCN-NH 2), a beta integrin antagonist, in patients with solid tumours. British journal of cancer 94 (11): 1621-1626. https://doi.org/10.1038/sj.bjc.6603171.

38. Amruta, N., Rahman, A., Pinteaux, E. and Bix, G. 2020. Neuroinflammation and fibrosis in stroke: the good, the bad and the ugly. Journal of Neuroimmunology 577318.

39. Narayanappa, A., Chastain, W.H., Paz, M., Solch, R.J., MurrayBrown, I.C., Befeler, J.B., Gressett, T.E., Longo, M.T., EnglerChiurazzi, E.B. and Bix, G. 2021. SARS-CoV-2 mediated neuroinflammation and the impact of COVID-19 in neurological disorders. Cytokine \& growth factor reviews.

40. Amruta, N., Engler-Chiurazzi, E.B., Murray-Brown, I.C., Gressett, T.E., Biose, I.J., Chastain, W.H. and Bix, G., 2021. In-vivo Protection from SARS-CoV-2 infection by ATN-161 in k18-hACE2 transgenic mice. bioRxiv. https://doi.org/10.1101/2021.05.08. 443275 .

41. Sui, A., Y. Zhong, A.M. Demetriades, Q. Lu, Y. Cai, Y. Gao, Y. Zhu, X. Shen, and B. Xie. 2018. Inhibition of integrin $\alpha 5 \beta 1$ ameliorates VEGF-induced retinal neovascularization and leakage by suppressing NLRP3 inflammasome signaling in a mouse model. Graefe's Archive for Clinical and Experimental Ophthalmology 256 (5): 951-961. https://doi.org/10.1007/s00417-018-3940-x.

42. Mortimer, L., F. Moreau, S. Cornick, and K. Chadee. 2015. The NLRP3 Inflammasome Is a Pathogen Sensor for Invasive Entamoeba histolytica via Activation of alpha5beta1 Integrin at the Macrophage-Amebae Intercellular Junction. PLoS Pathogens 11 (5): e1004887. https://doi.org/10.1371/journal.ppat.1004887.

43. St-Pierre, J., F. Moreau, S. Cornick, J. Quach, S. Begum, and L. Aracely Fernandez. 2017. The macrophage cytoskeleton acts as a contact sensor upon interaction with Entamoeba histolytica to trigger IL-1beta secretion. PLoS Pathogens 13 (8): e1006592. https:// doi.org/10.1371/journal.ppat.1006592. 
44. Kandikattu, H.K. 2018. Oxido-nitrosative stress and antioxidants in asthma. J Basic Clin Immonol 1: 9-12.

45. Chen, H.Y., L. Pan, H.L. Yang, P. Xia, W.C. Yu, W.Q. Tang, Y.X. Zhang, S.F. Chen, Y.Z. Xue, and L.X. Wang. 2018. Integrin alpha5 beta1 suppresses rBMSCs anoikis and promotes nitric oxide production. Biomedicine \& Pharmacotherapy 99: 1-8. https://doi.org/ 10.1016/j.biopha.2018.01.038.

46. Boveris, A., N. Oshino, and B. Chance. 1972. The cellular production of hydrogen peroxide. Biochemical Journal 128 (3): 617-630. https://doi.org/10.1042/bj1280617.

47. Nemoto, S., K. Takeda, Z.X. Yu, V.J. Ferrans, and T. Finkel. 2000 Role for mitochondrial oxidants as regulators of cellular metabolism. Molecular and cellular biology 20 (19): 7311-7318. https:// doi.org/10.1128/mcb.20.19.7311-7318.2000.

48. Nicholls, D.G., and S.L. Budd. 2000. Mitochondria and neuronal survival. Physiological reviews 80 (1): 315-360. https://doi.org/ 10.1152/physrev.2000.80.1.315.

49. Pinkse, G.G., W.P. Bouwman, R. Jiawan-Lalai, O.T. Terpstra, J.A. Bruijn, and E. de Heer. 2006. Integrin signaling via RGD peptides and anti- $\beta 1$ antibodies confers resistance to apoptosis in islets of Langerhans. Diabetes 55 (2): 312-317. https://doi.org/10.2337/ diabetes.55.02.06.db04-0195.

50. Werner, E., and Z. Werb. 2002. Integrins engage mitochondrial function for signal transduction by a mechanism dependent on Rho GTPases. The Journal of cell biology 158 (2): 357-368. https:// doi.org/10.1083/jcb.200111028.
51. Turečková, J., M. Vojtěchová, M. Krausová, E. Šloncová, and V. Korínek. 2009. Focal adhesion kinase functions as an akt downstream target in migration of colorectal cancer cells. Translational oncology 2 (4): 281-290. https://doi.org/10.1593/tlo.09160.

52. Caltagarone, J., Jing, Z. and Bowser, R. 2007. Focal adhesions regulate $A \beta$ signaling and cell death in Alzheimer's disease. Biochimica et Biophysica Acta (BBA)-Molecular Basis of Disease 1772(4):438-45. doi:https://doi.org/10.1016/j.bbadis.2006.11.007.

53. Reed, N.I., Jo, H., Chen, C., Tsujino, K., Arnold, T.D., DeGrado, W.F. and Sheppard, D. 2015. The $\alpha v \beta 1$ integrin plays a critical in vivo role in tissue fibrosis. Science translational medicine 7(288):288ra79. doi:https://doi.org/10.1126/scitranslmed.aaa5094.

54. Sen, T., A. Dutta, G. Maity, and A. Chatterjee. 2010. Fibronectin induces matrix metalloproteinase-9 (MMP-9) in human laryngeal carcinoma cells by involving multiple signaling pathways. Biochimie 92 (10): 1422-1434. https://doi.org/10.1016/j.biochi.2010. 07.005 .

55. González-Tarragó, V., A. Elosegui-Artola, E. Bazellières, R. Oria, C. Pérez-González, and P. Roca-Cusachs. 2017. Binding of ZO-1 to $\alpha 5 \beta 1$ integrins regulates the mechanical properties of $\alpha 5 \beta 1-$ fibronectin links. Molecular biology of the cell 28 (14): $1847-$ 1852. https://doi.org/10.1091/mbc.E17-01-0006.

Publisher's Note Springer Nature remains neutral with regard to jurisdictional claims in published maps and institutional affiliations. 